\title{
A systematic evaluation of yeast sample preparation protocols for spectral identifications, proteome coverage and post-isolation modifications
}

\author{
Maxime den Ridder, Ewout Knibbe, Wiebeke van den Brandeler, Pascale Daran-Lapujade and Martin Pabst* \\ Delft University of Technology, Department of Biotechnology, van der Maasweg 9, 2629 HZ Delft, The Netherlands
}

\begin{abstract}
The importance of obtaining comprehensive and accurate information from cellular proteomics experiments asks for a systematic investigation of sample preparation protocols, particularly when working with unicellular organisms with strong cell walls, such as found in the model organism and cell factory $\boldsymbol{S}$. cerevisiae. Sample preparation protocols may bias towards specific protein fractions or challenge the analysis of native protein modifications due to reagent-induced artefacts. Here, we performed a systematic comparison of sample preparation protocols using a matrix of different conditions commonly applied in whole cell lysate proteomics. The different protocols were evaluated for their overall fraction of identified spectra, proteome and amino acid sequence coverage, GO-term distribution and number of peptide modifications, by employing a combination of database and unrestricted modification search approaches. The best proteome and amino acid sequence coverage was achieved by using Urea combined with filter-aided or in-solution digestion protocols, where the overall outcomes were strongly influenced by the employed quenching procedure. Most importantly, the use of moderate incubation temperatures and times, circumvented excessive formation of modification artefacts. Extensive reagent-induced peptide modifications, however, were observed when using solvents such as acetone or additives such as formic acid. Moreover, several filter material-related modifications were observed when employing the filter-aided procedures. Ultimately, the best protocols enabled the identification of approximately $65-70 \%$ of all acquired fragmentation spectra, where additional de novo sequencing suggests that unidentified spectra were largely of too low spectral quality to provide confident spectrum matches. This study demonstrates the large impact of different sample preparation procedures on the proteomic analysis outcome, where the collected protocols and large sets of associated mass spectrometric raw data provide a resource to evaluate and design new protocols and guide the analysis of (native) peptide modifications in the model eukaryote yeast.
\end{abstract}

\section{INTRODUCTION}

Despite recent advancements in mass spectrometric instrumentation, a large fraction of fragmentation spectra (MS/MS) from bottom-up shotgun proteomics experiments usually remain unidentified $[1,2]$. Amongst the many possible reasons, a decreased identification rate can result from the presence of non-peptidic contaminants (particularly surfactants), increased spectral complexity due to co-fragmentation of multiple precursor ions, low-quality spectra due to poor and incomplete peptide fragmentation, unexpected peptide sequence variants or co/posttranslational modifications that are not covered by the database, as well as deficiencies of the database search scoring schemes used to match the spectra. The latter led to the development of iterative approaches or to the combined use of orthogonal database search algorithms [3-7]. However, a significant fraction of unassigned MS/MS spectra may also be a consequence of incomplete or nonspecific proteolytic cleavage, or result from unintended peptide modifications introduced during sample preparation when using highly reactive chemicals. In fact, sample preparation can be one of the most significant contributors to data variation and poor comparability between proteomics experiments [8]. Hence, selecting the most suitable sample preparation protocol is essential for
50 enabling a deep proteome coverage and for confidently 51 identifying and quantifying (novel) peptide modifications. 52 Moreover, the (native) in vivo state of the proteins should 53 be preserved as good as possible. Therefore, sample 54 preparation protocols usually include quenching of the 55 cellular metabolism subsequently after sampling. This is in 56 particular of importance, when investigating reversible 57 post-translational modifications, which may be rapidly 58 cleaved by the many hydrolytic enzymes present in every 59 cell [9]. However, different quenching strategies, such as 60 using ethanol or trichloroacetic acid (TCA) [10, 11], have 61 been shown to differently impact the outcome of proteomics 62 experiments [11]. Following protein extraction, proteins are 63 solubilised and commonly denatured (e.g., with Urea), 64 disulphide bonds are reduced by e.g., with Dithiothreitol 65 (DTT) or Tris(2-carboxyethyl)phosphine (TCEP), alkylated 66 using reagents such as iodoacetamide (IAA) or acrylamide 67 (AA) to avoid reoxidation of the sulfhydryl groups [12, 13] 68 and finally proteolytically cleaved (typically with trypsin) 69 to create protein fragments that are sufficiently small for 70 efficient measurement, but that retain sufficient unique 71 sequence information for the subsequent protein 72 identification step. Chemicals, or their employed 73 concentrations, are often not compatible with the following 74 steps in the protocol and, therefore, require removal by 
bioRxiv preprint doi: https://doi.org/10.1101/2022.01.17.476533; this version posted January 20, 2022. The copyright holder for this preprint (which was not certified by peer review) is the author/funder. All rights reserved. No reuse allowed without permission.

\section{Maxime den Ridder et al., 2022}

1 methods such as protein precipitation e.g., with acetone or

2 Trichloroacetic acid (TCA), or filter-aided approaches [143 20]. The type and $\mathrm{pH}$ of the buffer used during the final 4 proteolytic digestion may ultimately also impact the amino 5 acid cleavage site specificity [21]. Finally, non-peptidic 6 compounds and non-volatile salts are removed before 7 analysis by the aid of solid-phase extraction (SPE), using 8 different types of reverse-phase or mixed-mode resins [22].

9 However, combinations of different sample preparation 10 procedures are expected to differently bias the proteomic 11 analysis outcome. The selected protocol, therefore, may 12 influence the proteome fraction or native modifications that 13 can be detected and quantified significantly [23]. In addition, 14 when investigating the biological significance of peptide modifications, the observed modification needs to be traced back to its co/post-translational or sample processing origin. However, without appropriate controls, this is a highly challenging procedure. For example, formylation can occur as a natural histone modification [24], but may also be introduced during sample preparation when using formic acid-containing buffers, such as those used to increase the solubility of hydrophobic peptides $[25,26]$. Similarly for carbamylation, this modification has been associated with severe renal and cardiovascular disorders; however, it may also originate from high molarity Urea-containing buffers used during protein denaturation [27]. Alkylation of cysteine residues has become standard practice in proteomics experiments, which however due to over- or off-target alkylation reactions frequently introduces unintended peptide modifications, such as double alkylation artefacts, which for the case of IAA (114.04 Da) mimics the C-terminal glycine residue of ubiquitin [12, 13, 28, 29]. Nevertheless, recent studies suggest that disulphide reduction and cysteine alkylation may not be essential to achieve a good proteome coverage. This would therefore eliminate the exposure of the peptides to highly reactive alkylating reagents [30]. Furthermore, chemically labile amino acid residues may undergo sample preparation induced oxidation, deamidation, pyroglutamate formation, dehydration or excessive metal ion adduct formation [31-33]. Many of those modifications (or adducts) may, however, also occur as consequence of the natural protein ageing processes within the cell and are, therefore, difficult to discriminate from sample preparation artefacts [34].

A systematic evaluation - using a matrix-like approach, which leaves out one chemical at a time - and which investigates the impact on i) the $\%$ of identifications, ii) proteome coverage, iii) GO-term distribution and iv) post-isolation modifications, has not been performed for an unicellular organism to date. We therefore constructed a matrix of conditions, which include steps frequently employed in whole cell lysate proteomics, and applied it to the preparation of well-controlled chemostat grown yeast cells. The outcomes of the proteomics experiments were compared for their obtained proteome and amino acid sequence coverage and for their GO-term profiles. Moreover, we performed an unrestricted modification search using TagGraph, to identify reagent-induced modifications. Finally, we investigated the quality of the unidentified spectra using de novo peptide sequencing. In summary, this study provides a systematic evaluation of sample preparation protocols for the increasingly studied model organism and cell factory yeast. Thereby, we demonstrate the large impact of the different sample preparation procedures on proteome coverage and overall identification rates.

Ultimately, the performed study and publicly available large proteomics datasets, provide a valuable resource to select for the most suitable sample preparation elements for different experiments, and support the analysis of (native) peptide modifications.

\section{EXPERIMENTAL SECTION}

Yeast strain, growth media and storage. In this study, we used the minimal glycolysis (MG) yeast strain IMX372 (MATa ura3-52 his3-1 leu2-3,112 MAL2-8c SUC2 glk1::SpHis5, hxk1::KlLEU2, tdh1::KlURA3, tdh2, gpm2, gpm3, eno1, pyk2, pdc5, pdc6, adh2, adh5, adh4), which under the selected growth conditions shows now phenotypic alterations compared to the parent CEN.PK lineage [35, 36]. Shake flask and chemostat cultures were grown in synthetic medium (SM) containing $5.0 \mathrm{~g} \cdot \mathrm{L}^{-1}(\mathrm{NH} 4)_{2} \mathrm{SO}_{4}, 3.0 \mathrm{~g} \cdot \mathrm{L}^{-1}$ $\mathrm{KH}_{2} \mathrm{PO}_{4}, 0.5 \mathrm{~g} \cdot \mathrm{L}^{-1} \quad \mathrm{MgSO}_{4} \cdot 7 \mathrm{H}_{2} \mathrm{O}$ and $1 \mathrm{~mL} \cdot \mathrm{L}^{-1}$ trace elements in demineralized water. The medium was heat sterilized $\left(120^{\circ} \mathrm{C}\right)$ and supplemented with $1 \mathrm{~mL} \cdot \mathrm{L}^{-1}$ filter sterilized vitamin solution [37]. For shake flask cultures 20 $\mathrm{g} \cdot \mathrm{L}^{-1}$ heat sterilized $\left(110^{\circ} \mathrm{C}\right)$ glucose $(\mathrm{SMG})$ was added. In chemostat cultures, $7.5 \mathrm{~g} \cdot \mathrm{L}^{-1}$ glucose was added and the medium was supplemented with $0.2 \mathrm{~g} \cdot \mathrm{L}^{-1}$ antifoam Pluronic PE 6100 (BASF, Ludwigshafen, Germany). Frozen stocks of $S$. cerevisiae cultures were prepared by the addition of glycerol $(30 \% \mathrm{v} / \mathrm{v})$ in $1 \mathrm{~mL}$ aliquots for storing at $-80^{\circ} \mathrm{C}$. Yeast chemostat cultures and sampling. Aerobic shake flask cultures were grown at $30^{\circ} \mathrm{C}$ in an Innova incubator shaker (New Brunswick ${ }^{\mathrm{TM}}$ Scientific, Edison, NJ, USA) at $200 \mathrm{rpm}$ using $500 \mathrm{~mL}$ round-bottom shake flasks containing $100 \mathrm{~mL}$ medium. Duplicate aerobic chemostat cultures were performed in 2 L laboratory fermenters (Applikon, Schiedam, The Netherlands) with a $1 \mathrm{~L}$ working volume in duplicate. SM-medium was used and maintained at $\mathrm{pH} 5$ by the automatic addition of $2 \mathrm{M} \mathrm{KOH}$. Mixing of the medium was performed with stirring at $800 \mathrm{rpm}$. Gas inflow was filter sterilized and compressed air (Linde Gas, Schiedam, The Netherlands) was sparged to the bottom of the bioreactor at a rate of $500 \mathrm{~mL} \cdot \mathrm{min}^{-1}$. Dissolved oxygen levels were measured with Clark electrodes (Mettler Toledo, Greinfensee, Switzerland). The temperature of the fermenters was maintained at $30^{\circ} \mathrm{C}$. The reactors were inoculated with exponentially growing shake flask cultures 
1 of $S$. cerevisiae strain IMX372 to obtain an initial optical

2 density $\left(\mathrm{OD}_{660}\right)$ of approximately 0.4 . Following the batch

3 phase, the medium pump was switched on to obtain a

4 constant dilution rate of $0.10 \mathrm{~h}^{-1}$. Chemostat cultures were

5 assumed to be in steady state when, after five volume

6 changes, the culture dry weight, oxygen uptake rate and $\mathrm{CO}_{2}$

7 production rate varied less than $5 \%$ over at least 2 volume

8 changes. The dilution rate and carbon recovery were

9 determined after each experiment. Analytical methods.

$10 \mathrm{OD}_{660}$ measurements to monitor growth were performed on

11 a JENWAY 7200 spectrophotometer (Cole-Parmer, Stone,

$12 \mathrm{UK})$. The biomass dry weight was determined in duplicate

13 by extracting $10 \mathrm{~mL}$ of broth and filtrating it over a filter

14 with $0.45 \mu \mathrm{m} \varnothing$ pores while a vacuum was applied to the

15 filter. The filters were washed twice with $10 \mathrm{~mL}$ of

16 demineralized water. Prior to use, the filters were dried in

17 the oven for at least 24 hours at $70^{\circ} \mathrm{C}$. After filtration, the

18 filters were dried in a microwave oven at $360 \mathrm{~W}$ for $20 \mathrm{~min}$

19 leaving only dry biomass. For extracellular metabolite

20 determinations, broth samples were centrifuged for $5 \mathrm{~min}$ at

$2113.000 \mathrm{~g}$ and the supernatant was collected for subjection to

22 a Waters alliance 2695 HPLC (Waters Chromatography

23 B.V., Etten-Leur, The Netherlands) with an Animex HPX-

$2487 \mathrm{H}$ ion exchange column (Bio-Rad, Hercules, CA, USA).

25 The HPLC was operated at $60^{\circ} \mathrm{C}$ and $5 \mathrm{mM}$ of $\mathrm{H}_{2} \mathrm{SO}_{4}$ was

26 used as mobile phase at a rate of $0.6 \mathrm{~mL} \cdot \mathrm{min}^{-1}$. Off-gas

27 concentrations of $\mathrm{CO}_{2}$ and $\mathrm{O}_{2}$ were measured using an NGA

282000 analyzer. Proteome samples $\left(1 \mathrm{~mL}\right.$, at approx. $3.6 \mathrm{~g} \cdot \mathrm{L}^{-}$

$29{ }^{1}$ dry weight) were taken from steady state cultures. The

30 samples were collected in multifold in trichloroacetic acid

31 (TCA) (Merck Sigma, Cat. No. T0699) with a final

32 concentration of $10 \%$ or in five volumes of ice-cold

33 methanol (MeOH) (Thermo Fisher, Cat. No. 15654570).

34 Samples were centrifuged at $4000 \mathrm{~g}$ for $5 \mathrm{~min}$ at $4^{\circ} \mathrm{C}$. Cell

35 pellets were frozen at $-80^{\circ} \mathrm{C}$ [11]. Proteomics sample

36 preparation protocols (an extended version of all sample

37 preparation protocols are provided in the SI document).

38 Yeast cell culture pellets were resuspended in lysis buffer

39 composed of $100 \mathrm{mM}$ triethylammonium bicarbonate

40 (TEAB) (Merck Sigma, Cat. No. T7408) containing 0.1\%,

$411 \%$ sodium dodecyl sulphate (SDS) (Merck Sigma, Cat. No.

42 L4522) or 8 M Urea (Merck Sigma, Cat. No. U5378) and

43 phosphatase/protease inhibitors. Yeast cells were lysed by

44 glass bead beating and thus shaken 10 times for 1 minute

45 with a bead beater alternated with 1 minute rest on ice. For

46 in-solution methods, proteins were reduced by addition of 5

47 mM DTT (Merck Sigma, Cat. No. 43815) or 5 mM TCEP

48 (Merck Sigma, Cat. No. C4706) and incubated for 1 hour or

$4930 \mathrm{~min}$ at $37^{\circ} \mathrm{C}$ or $56^{\circ} \mathrm{C}$, respectively. Subsequently, the

50 proteins were alkylated for $30 \mathrm{~min}$ or 1 hour at room

51 temperature in the dark by addition of $15 \mathrm{mM}$
52 iodoacetamide (Merck Sigma, I1149) or $50 \mathrm{mM}$ acrylamide (AA) (Merck Sigma, Cat. No. A9099), respectively. Protein precipitation was performed by addition of four volumes of ice-cold acetone $\left(-20^{\circ} \mathrm{C}\right)$ (Merck Sigma, Cat. No. 650501) or TCA to a final concentration of $20 \%$ and proceeded for 1 hour at $-20^{\circ} \mathrm{C}$ or $30 \mathrm{~min}$ at $4^{\circ} \mathrm{C}$, respectively. The proteins were washed twice with acetone and subsequently solubilized using $100 \mathrm{mM}$ ammonium bicarbonate (ABC) (Merck Sigma, Cat. No. 09830). Alternatively, for filteraided sample preparation (FASP), proteins were loaded to a filter (Merck-Millipore, Microcon $10 \mathrm{kDa}$, Cat. No. MRCPRT010) after bead beating and reduced by addition of DTT and alkylated with iodoacetamide, as described earlier. After alkylation, proteins were washed four times with TEAB and ABC buffers. For all protocols, proteolytic digestion was performed by Trypsin (Promega, Cat. No. V5111), 1:100 enzyme to protein ratio (v/v), and incubated at $37^{\circ} \mathrm{C}$ overnight. For filter-aided sample preparation protocols, peptides were eluted from the filters after digestion using $\mathrm{ABC}$ and 5\% acetonitrile (ACN) (Thermo Fisher, Cat. No. 10489553) / 0.1\% formic acid (FA) (Thermo Fisher, Cat. No. 10596814) buffers consecutively. For all protocols, solid phase extraction was performed with an Oasis HLB 96-well $\mu$ Elution plate (Waters, Milford, USA, Cat. No. 186001828BA). Peptide fractions were eluted using $\mathrm{MeOH}$ buffer containing trifluoroacetic acid (TFA) (Merck Sigma, Cat. No. 302031), FA or ABC. Eluates were dried using a SpeedVac vacuum concentrator. Dried peptides were resuspended in 3\% ACN / 0.01\% TFA prior to MS-analysis to give an approximate concentration of $500 \mathrm{ng}$ per $\mu \mathrm{L}$. Shotgun proteomic analysis. For each protocol an aliquot corresponding to approx. $750 \mathrm{ng}$ protein digest was analysed using a one-dimensional shotgun proteomics approach [38]. Each sample was analysed in two technical replicates. Briefly, the samples were analysed using a nano-liquid-chromatography system consisting of an EASY nano LC 1200, equipped with an Acclaim PepMap RSLC RP C18 separation column (50 $\mu \mathrm{m}$ x 150 $\mathrm{mm}, 2 \mu \mathrm{m}$, Cat. No. 164568), and a QE plus Orbitrap mass spectrometer (Thermo Fisher Scientific, Germany). The flow rate was maintained at $350 \mathrm{~nL} \cdot \mathrm{min}^{-1}$ over a linear gradient from $5 \%$ to $30 \%$ solvent B over $90 \mathrm{~min}$, then from $30 \%$ to $60 \%$ over $25 \mathrm{~min}$, followed by back equilibration to starting conditions. Data were acquired from 5 to $120 \mathrm{~min}$. Solvent $\mathrm{A}$ was $\mathrm{H}_{2} \mathrm{O}$ containing $0.1 \% \mathrm{FA}$, and solvent $\mathrm{B}$ consisted of $80 \% \mathrm{ACN}$ in $\mathrm{H}_{2} \mathrm{O}$ and $0.1 \%$ FA. The Orbitrap was operated in data-dependent acquisition (DDA) mode acquiring peptide signals from $385-1250 \mathrm{~m} / \mathrm{z}$ at 70,000 resolution in full MS mode with a maximum ion injection time (IT) of $100 \mathrm{~ms}$ and an automatic gain control (AGC) target of 3E6. The top 10 precursors were selected for 
1 MS/MS analysis and subjected to fragmentation using

2 higher-energy collisional dissociation (HCD). MS/MS

3 scans were acquired at 17,500 resolution with AGC target

4 of $2 \mathrm{E} 5$ and IT of $75 \mathrm{~ms}, 2.0 \mathrm{~m} / \mathrm{z}$ isolation width and

5 normalized collision energy (NCE) of 28. Mass

6 spectrometric raw data processing. De novo sequence

7 analysis. De novo sequencing was performed using the

8 algorithm available via PEAKS Studio $\mathrm{X}+$ (Bioinformatics

9 Solutions Inc., Waterloo, Canada) [39], allowing 10 ppm

10 parent ion and $0.5 \mathrm{Da}$ fragment ion mass error, and

11 oxidation as variable modification, where the resulting de

12 novo sequences were exported to 'de novo peptide.csv' files

13 for further unrestricted modification search using

14 TagGraph, as described below. Taxonomic profiling.

15 Taxonomic purity assessment using the same de novo

16 peptide sequences was performed as described recently by

17 H.B.C. Kleikamp et al. (2021) [40]. Database searching.

18 Database searching against the proteome database from $S$.

19 cerevisiae (Uniprot, strain ATCC 204508 / S288C, Tax ID:

20 559292, June 2020, excluding 13 glycolytic isoenzymes)

21 was performed using PEAKS Studio $\mathrm{X}+$, allowing for 20

$22 \mathrm{ppm}$ parent ion and $0.02 \mathrm{~m} / \mathrm{z}$ fragment ion mass error, 3

23 missed cleavages, carbamidomethyl or acrylamide as fixed

24 (or none), and methionine oxidation and N/Q deamidation

25 as variable modifications. To control false-positive peptide

26 identifications, a uniform 1\% false discovery rate (FDR)

27 was applied to peptide spectrum matches (PSM), and

28 subsequently the protein identifications required $\geq 2$ unique

29 peptides. Results from the PEAKS DB search were

30 exported to 'proteins.csv' and 'DB search psm.csv' files,

31 containing the identified proteins and identified DB search

32 peptide-spectrum matches, respectively. Unrestricted

33 modification search. TagGraph [41] was used to perform

34 unrestricted global peptide modification search using the

35 (mzML-formatted) mass spectrometric raw data and the de

36 novo sequences obtained from PEAKS Studio, using the

37 yeast proteome database plus the CRAPome contaminant

38 sequences [42]. The analysis was performed allowing for 10

39 ppm precursor mass tolerance, cysteine

40 carbamidomethylation or acrylamidation as static

41 modifications, and methionine oxidation as a differential

42 modification, as described by Devabhaktuni et al. (2019)

43 [41]. TagGraph.1.8 was installed on a Windows desktop

44 Docker container, and the processing of multiple files was

45 automated via a PowerShell script. An FDR of 1\%, 10 ppm

46 parent ion and a maximum absolute deviation of $0.1 \mathrm{Da}$

47 between experimental and database modification mass were

48 applied to the analysis results and these were exported to

49 '.txt' files for further analysis. Interconversion of mass

50 spectrometric raw data. Conversion of the mass

51 spectrometric raw data was performed using peak picking
52 'vendor' into '.mzML' and '.mzXML' using the 53 msConvertGUI tool (ProteoWizard) [43]. Identification of 54 scans originating from glycopeptides. First, the 55 fragmentation spectra were searched for the presence of 56 glycan-typical HexNAc oxonium fragment ions (204.087, $\left.57\left[\mathrm{M}+\mathrm{H}^{+}\right]^{+}\right)$using functions from the Matlab 'sugar miner' 58 script as described recently [44]. Those scans indicate 59 glycopeptides-which remain unidentified by the chosen 60 database, or open modification search parameters. Scans 61 with strong oxonium ion signals were summarized in an 62 '.xlsx' table. Data processing and visualization. Overall 63 number of modified peptides and types of modifications.

64 The results of the unrestricted modification search using

65 TagGraph were used to determine the overall volume as

66 well as the types of modifications found after applying the

67 different protocols. First, the mass shifts (=deviations from

68 the unmodified peptide mass) were collected from the ".txt"

69 files and binned using the Matlab 'histcounts' function, at a

70 bin width of $0.01 \mathrm{Da}$. This procedure was done for the

71 combined dataset as well as for each protocol separately.

72 Mass shifts with more than 5 occurrences per averaged

73 conditions were tested for significant changes across all

74 protocols using Matlabs 'anova1' function. Mass shifts with

75 significant changes $(\mathrm{p}<0.01)$ across all conditions (2

76 biological and 2 technical replicates) were visualized by

77 Euclidean distance clustering using the Matlab

78 'clustergram' function, standardizing along the rows (mass

79 shifts) of data, clustering along the columns of data, and

80 then clustering along the rows of row-clustered data.

81 Default color variation has been used which shows for

82 values between -3 and 3 , where values above and below

83 show the same maximum color tone. Spectral Average

84 Local Confidence (ALC) score histograms. After the scan

85 numbers of each protocol were allocated to different

86 categories, the Average Local Confidence (ALC) score

87 distribution for identified and unidentified MS/MS scans

88 was determined to evaluate the quality of the unidentified

89 spectra. The ALC score was extracted from the de novo

90 sequences '.csv' files and were therefore only determined

91 for the spectra that were de novo sequenced. The ALC

92 scores of the identified and unidentified scans were binned

93 separately using the 'histcounts' function in Matlab. The

94 resulting distributions were plotted as bar graph and

95 exported as table. Proteome and amino acid sequence

96 coverage. The proteome coverage was determined using the

97 PEAKS-DB search results 'proteins.csv'. For this, the

98 number of proteins per protocol was calculated from

99 proteins with $>2$ unique peptides per protein. The

100 percentage of observed proteins for each protocol was

101 subsequently calculated by normalizing to the number of

102 total proteins in the S. cerevisiae (Uniprot) protein sequence 
1 database. The average protein sequence coverage per

2 protocol was further extracted from the PEAKS

3 'proteins.csv' output files. The sequence coverage (\%) was

4 moreover used to determine the amino acid coverage, in

5 which the sequence coverage of each identified protein was

6 summed and related to the total number of protein

7 sequences present in the sequence database. The average for

8 the proteome and amino acid coverage was calculated for

9 each biological replicate and the deviations from the

10 average for each protocol were plotted as bar graphs. A two-

11 tailed unpaired Student's t-test was performed to determine

12 if the relative coverage change between protocols was

13 statistically significant. Ontology analysis. The

14 'proteins.csv' files obtained through PEAKS-DB were used

15 to determine the differences in the cellular component

16 distribution. Python 3.8 [45] was used to programmatically

17 link the Uniprot accession numbers of the identified

18 proteins (with 2 unique peptides) to the Gene Ontology

19 (GO) [46] terms using Retrieve/ID Mapping function on

20 Uniprot [47]. The GO library was imported using the

21 'goatools' module in Python to retrieve the cellular

22 component terms in the GO hierarchy. Functions of the

23 proteins were summarized in pie charts and bar graphs

24 based on their cellular component GO terms. GO terms with

25 less than one percentage contribution were clustered into

26 category "other". Overall \% of identified fragmentation

27 spectra. The overall number of identified fragmentation

28 (MS/MS) spectra for each protocol was determined by

29 combining the outputs obtained from TagGraph, PEAKS

30 database search and the sugar oxonium ion search. First,

31 scan numbers that did not result into amino acid sequence

32 candidates by PEAKS de novo sequencing were allocated to

33 the 'No ALC' category. Next, identifications were extracted

34 from the TagGraph files. Thereby, identified scans were

35 allocated to two categories, 'unmodified peptides' and

36 'modified peptides '. The scan numbers of the peptides

37 identified with the second search engine were extracted

38 from the PEAKS-DB search peptide-spectrum matches files

39 'DB search psm.csv'. The scan numbers were compared

40 with the scans of the de novo sequences and TagGraph

41 results. Identifications that were only found with PEAKS

42 database search, were allocated to the 'second search

43 engine' category. The category "sugars" represents the

44 scans that were identified as potential glycopeptides. If

45 scans were present in multiple categories, including

46 TagGraph, the scans were allocated to TagGraph

47 identifications (modified or unmodified peptides). If scans

48 were identified containing sugar fragments and by PEAKS

49 database search, the scans were allocated to PEAKS-DB

50 search identifications. Furthermore, 'No ALC'-allocated

51 scans that could be identified containing sugar fragments or
52 via PEAKS database search, were allocated to one of the

53 respective categories. Finally, the MS/MS scans that could

54 not be identified by any of the before mentioned categories,

55 were allocated to the 'unidentified scans' category. As final

56 check, the summed scans of all established categories

57 required to be equal to the sum of MS/MS scans in the raw

$58 \mathrm{mzXML}$ files. The distribution of the scan identifications

59 amongst different categories was visualized using stacked

60 bar graphs, in which the number of scans in each category

61 was normalized against the total number of MS/MS scans

62 in percentages, for each protocol. Data availability. Mass

63 spectrometric raw data, protein sequence database, and

64 search files have been deposited at ProteomeXchange

65 server and are publicly available under the project code

66 PXD026806.

67

\section{RESULTS AND DISCUSSION}

69 Comparison of yeast whole cell lysate sample

70 preparation protocols. A systematic comparison of a matrix

71 of different sample preparation procedures was performed to

72 investigate the impact on spectral identification rates and

73 quality, achieved proteome coverage, amino acid sequence

74 coverage, GO-terms distribution and reagent-induced peptide

75 modifications (Table 1, Figure 1). Duplicate aerobic chemostat

76 cultures of the IMX372 S. cerevisiae strain [36] were cultured

77 in glucose-limiting conditions (Supplementary Table S1),

78 which provided highly reproducible yeast cell biomass.

79 Proteome samples were taken during steady-state conditions

80 from both biological replicates, in which the cellular

81 metabolism was quenched using ice-cold TCA or methanol to

82 preserve the proteome and associated post-translational

83 modifications. Cell lysis was performed with SDS- or Urea-

84 containing buffers employing bead-beating in all cases. Crude

85 cell lysates were then treated with an in-solution or FASP

86 approach, in which proteins were reduced with TCEP or DTT

87 and alkylated with iodoacetamide, acrylamide or alternately,

88 were left untreated. For in-solution digestion methods, proteins

89 were precipitated with acetone or TCA. After overnight

90 digestion with trypsin, solid-phase extraction was performed to

91 remove contaminants by SPE using Oasis HLB cartridges,

92 which make use of a co-polymer of divinylbenzene and vinyl

93 pyrrolidinone that shows an enhanced retention of polar

94 peptides (Waters). Elution from the cartridges was achieved

95 with a variety of buffers to assess the influence of different

96 additives (Table 1). Finally, the samples of the different

97 protocols were analysed using a (short) one-dimensional

98 gradient with duplicate injections.

99 On average, $35420 \pm 5909$ (biological replicate 1) and

$10037261 \pm 8271$ (biological replicate 2) MS/MS scans were 101 obtained across all protocols (Figure 2, Supplemental Figure

102 S1). Furthermore, the analysis of the protocols resulted in

$10323631 \pm 5237$ and $23477 \pm 6590$ peptide spectrum matches

104 (PSMs) (Figure 2a), resulting in 66\% and 61\% MS/MS 
1 spectrum identifications on average (Figure 2b). However, 2 protocols 8 and 14 resulted consistently in a considerable lower 3 number of MS/MS spectra for both biological replicates, albeit 4 that a comparable amount of proteolytic digest (750 ng) was 5 analysed. For protocol 8, protein precipitation was performed 6 with TCA/acetone instead of acetone solely. Difficulties in re7 dissolving the protein pellet can arise for the TCA/acetone 8 precipitation approach and may ultimately impact the overall 9 recovery. It therefore becomes necessary to use stronger 10 buffers, larger volumes and additional mechanical disruption 11 of the pellet, to solubilise the protein $[15,18,48]$. In this study, 12 protein pellets were dissolved in ammonium bicarbonate 13 buffers to allow subsequent trypsin digestion, which did not 14 prove difficult for acetone-precipitated samples. However, the 15 TCA/acetone procedure led to a partially insoluble pellet, and 16 a considerably lower number of proteins were in the following 17 identified, compared to when only using acetone-precipitation $18(20 \%$ vs. $29 \%$ proteome coverage for protocol $8=$

19 TCA/acetone, vs protocol $7=$ acetone; Figure 2c). Similar 20 results were observed in other studies, which was attributed to 21 the increased protein denaturation caused by this approach [15, 22 49] [50] [51]. Samples treated according to protocol 14 were 23 subjected to FASP using an SDS containing buffer. SDS is 24 known to interfere with binding and elution during the reverse25 phase separation of the peptides and severely suppresses 26 ionisation by electrospray ionisation [52-54]. Multiple rounds 27 of centrifugation were applied to ensure SDS removal, 28 however, residual SDS may have still impacted the LC-MS/MS 29 analysis, resulting in a lower total number of MS/MS scans 30 (13232 MS/MS spectra on average for protocol 14, vs. 36340 $31 \mathrm{MS} / \mathrm{MS}$ spectra on average across all protocols). However, an 32 earlier study buffer exchanged SDS with Urea following 33 solubilisation of the proteins, allowing for high protein 34 identification rates, because SDS was likely successfully 35 withdrawn from the sample [19]. Hence, FASP sample 36 preparation in combination with SDS-containing buffers is not 37 recommended unless the complete removal of SDS can be 38 ensured.

40 Overall proteome coverage is strongly affected by 41 sample preparation procedures. MS/MS spectra were identified using database searching employing common statistical filtering criteria (see materials and methods) and requiring at least 2 unique peptides per protein identification. The proteome coverage was calculated based on the proteins identified per protocol as a percentage of the total number of proteins in yeast (known ORFs). An average proteome coverage of approximately $26 \%$ and $27 \%$ was achieved amongst the different methods for biological replicates 1 and 2, respectively (Figure 2c). Small differences in the average depth of the proteome coverage between both biological replicates were observed, most likely because the experiments were conducted at different time periods and, therefore, the instrumental performance may have been slightly different. Three protocols $(6,8$ and 14) differed substantially from the other procedures. As discussed above, for protocols 8 and 14, LC-MS/MS analysis was likely compromised by incompatibility with the used reagents. Protocol 6, on the other hand, resulted in an average number of $39152 \mathrm{MS} / \mathrm{MS}$ spectra, which is slightly higher than the average of $36341 \mathrm{MS} / \mathrm{MS}$ spectra across all protocols (Figure 2b). Since the total ion count was very comparable, its suggestive that a similar amount of proteolytic digest was injected to the LC-MS system. This was the only sample that was subjected to quenching with methanol as opposed to TCA during sampling. Methanol quenching (applied to yeast) might, therefore, significantly impact the number of identifiable proteins. TCA as a quenching solution has been also proven useful in quantitative phosphoproteomics measurements recently [11]. Methanol, on the other hand, is routinely employed to rapidly arrest the cellular metabolism when performing metabolomics studies [55]. Methanol has been also used to co-extract metabolites and proteins from yeast, only recently [56]. In the present study, the methanol quenching bias was observed consistently for all biological and technical replicates. Protein aggregation due to exposure to methanol followed by poor resolubilisation is likely the cause for the low proteome coverage, however, the exact mechanism remains to be explored.

Nonetheless, the highest proteome coverage (31\% and 32\%, for biological replicates 1 and 2, respectively) was obtained with sample 13, in which a filter-aided approach was used in combination with Urea buffer. Similar results have been found in previous studies, in which FASP outperformed in-solution approaches in terms of the number of protein identifications $[57,58]$.

The proteome coverage does not seem to have been significantly impacted by the type of detergent used during lysis (SDS or Urea) for samples 7 and 12, respectively. Typically, SDS is used as an ionic and denaturing surfactant that can disrupt cell membranes and cause protein denaturation by disrupting protein-protein interactions. Urea, on the other hand, is a chaotrope that can bind to proteins, thereby causing protein unfolding [59]. Following protein extraction, protein reduction was performed by either TCEP or DTT. The type of reducing agent used during sample processing was also not a key determinant in the outcome of the proteome analysis. This was very similarly observed for yeast in another study [13]. In this study, proteins were subsequently alkylated with iodoacetamide, acrylamide or alkylation was left out completely (samples 7, 9 and 10, respectively). Expectedly, and in line with a recent publication [30], the absence of an alkylation step dramatically reduced the detection of cysteinecontaining peptides, although the depth of the proteome coverage remained nearly unchanged. However, the number of 
1 MS/MS scans and obtained peptide spectrum matches, as well

2 as sequence coverage, was steadily lower for the non-alkylated

3 sample (protocol 10), when compared to the alkylated samples

4 (protocols 7 and 9). Furthermore, no significant changes in the

5 number of identified proteins could be observed for the

6 different solvents used for solid-phase extraction (protocols 1-

7 5, 7, Figure 2 and Supplemental Table S2 and S3). Though, the

8 number of MS/MS scans, identified proteins and proteome

9 (sequence) coverage was consistently higher for protocol 7

10 (Figure 2, Supplemental Table S2 and S3), in which a

11 combination of basic and acidic $\mathrm{MeOH}$ buffers were used for

12 elution, thereby maximising the recovery of peptides across a

13 large $\mathrm{pH}$ range. Moreover, TFA (protocol 7) appeared to be a

14 better choice for peptide elution compared to formic acid (FA)

15 (protocol 2 and 5) because the proteome (sequence) coverage

16 was repeatedly higher when using TFA compared to FA

17 (Supplemental Table S2 and S3).

18 A gene ontology (GO) analysis [46] was performed to

19 investigate whether the different protocols bias towards

20 specific cellular components, such as cytosolic soluble

21 proteins, or oppositely, towards hydrophobic membrane

22 proteins. Therefore, GO terms were assigned to proteins based

23 on their 'cellular component'. Thereby, the distribution of the

24 cellular localisation of the observed proteins was comparable

25 across the different protocols, which is exemplified in Figure

26 3a for protocol 13 (biological replicate 2, Supplemental Table

27 S4). Approximately 53\% accounted for the intracellular

28 fraction (GO-term 'organelle'), whereas $\sim 11 \%$ of the

29 identifications belonged to membrane proteins. Yet, a slight

30 increase in the average relative value of membrane proteins

31 was observed for protocols 8 and 14 (Figure 3b). Irrespective

32 of the protocol, proteins were extracted using bead beating,

33 which is a relatively harsh and likely reproducible approach.

34 Nevertheless, a recent study demonstrated that extraction

35 methods can significantly contribute to the variability of

36 proteomics experiments [8].

37 Commonly, proteins are already reported with a few

38 peptides, e.g. 2 unique peptides. While acceptable for

39 qualitative and quantitative analysis, further

40 characterisation of individual proteoforms as well as

41 analysis of post-translational modifications demand a

42 higher sequence coverage. Therefore, we quantified the

43 fraction of the total proteome amino acid sequence space

44 covered for every protocol. An average proteome sequence

45 coverage of $6.7 \%$ and $7.0 \%$ was observed across all

46 protocols for biological replicates 1 and 2, respectively

47 (Figure 2d). Hence, for the employed (relatively short) one-

48 dimensional gradient-despite the good proteome

49 coverage - a rather small fraction of the amino acid

50 sequence space was actually observed in our experiments.

51 In the present study, an average protein sequence of $26 \%$

52 and $25 \%$ was obtained amongst the identified proteins

53 (Supplemental Figure S2). This value is proportional to the
54 average amino acid sequence coverage of $28 \%$ obtained by

55 Herbert et al. (2014), who obtained through extensive

56 experiments a near-complete proteome coverage [61].

57 Nevertheless, the proteome coverage strongly depends on

58 the protein amino acid sequences, and therefore, on the

59 cleavage specificity of the employed protease(s) [60].

60 One of the challenges to improve proteome sequence

61 coverage arises from signal suppression during electrospray

62 ionisation (particularly for short one-dimensional

63 gradients), where peptides with higher basicity tend to

64 ionise preferably, and lower abundant peptides or highly

65 hydrophobic or acidic peptides may remain undetected.

66 Longer LC separations, additional peptide pre-

67 fractionation, as well as 2-dimensional gradients

68 significantly increases the number of identifications [62]

69 [63-65]. Nevertheless, material requirements, sample

70 processing and analysis time will increase proportionally,

71 when using multi-dimensional chromatography or off-line

72 peptide fractionation. Finally, multi-protease digestion has

73 been demonstrated to boost not only the protein but also the

74 proteome sequence coverage [66, 67].

75 As observed for the proteome coverage, protocols 6, 8 and

7614 resulted in a much lower proteome sequence coverage

77 (Figure 2d, Supplemental Table S2 and S3). Overall, while

78 lysis buffer, reducing agent or SPE elution buffers

79 seemingly did not impact proteome coverage, the alkylation

80 step was critical for obtaining a high amino acid sequence

81 coverage.

82 The observed variability in peptide modifications. By

83 using an unrestricted modification search (TagGraph)

84221751 and 181496 mass shifts were observed across all

85 used protocols, which derived from 991759 and 1043314

86 MS/MS spectra for biological replicates 1 and 2,

87 respectively (Figure 4). A highly comparable mass shift

88 profile was observed for both technical and biological

89 replicates, confirming the reproducibility of the chemostat

90 cultivation [68], the sample preparation protocols and the

91 shotgun analysis. To discriminate between peptide

92 modifications from biological (co/post-translational) and

93 sample processing origin, we searched for alterations (or

94 mass shifts) that were predominantly (or exclusively)

95 present when using certain sample preparation protocols.

96 Hence, we searched for mass shifts that showed a significant

97 change in frequency $(\mathrm{p}<0.01)$ across all conditions and

98 replicates, of which the averaged occurrences across all

99 protocols is shown in Figure 5 (Supplementary Table S5).

100 Albeit we employed combinations of well-established

101 sample preparation steps, we observed a distinct number of

102 condition-specific modifications, of which some had not been

103 linked to sample preparation artefacts before. 
bioRxiv preprint doi: https://doi.org/10.1101/2022.01.17.476533; this version posted January 20, 2022. The copyright holder for this preprint (which was not certified by peer review) is the author/funder. All rights reserved. No reuse allowed without permission.

\section{Maxime den Ridder et al., 2022}

1 For example, two different approaches to quench the cellular 2 metabolism were employed, using either TCA or methanol. A 3 mass shift of $-89.04 \mathrm{Da}$ was prominent in TCA-arrested yeast 4 cells, with the notable exception of protocol 14 (SDS/FASP 5 protocol). Furthermore, we used reagents for cell lysis and 6 protein denaturation such as SDS and Urea. Interestingly, we 7 did not observe any mass shifts related to the use of those 8 reagents. In aqueous solutions, Urea dissociates upon heating 9 over time. One of its degradation products is iso-cyanate, 10 which can react with the N-termini of proteins/peptides and at 11 the side-chain amino groups of lysine and arginine residues, to 12 mimic in vivo carbamylation $(+43 \mathrm{Da})$. This reaction is 13 enhanced by high temperatures; however, Urea solutions in our 14 study were only heated to $37^{\circ} \mathrm{C}$ for 1 hour, whereas $56^{\circ} \mathrm{C}$ is 15 commonly used for protein reduction with DTT or TCEP [69].

16 Furthermore, the use of ammonium-containing buffers (and the

17 removal of Urea before over-night digestion) seemed to

18 minimise protein carbamylation when using Urea solutions

19 [70], which was also observed in our study. Therefore, the

20 relatively mild procedure used by our protocols seemingly

21 avoided carbamylation reactions taking place.

22 No significant mass shifts were observed between samples

23 reduced with TCEP or DTT. This is in line with an earlier study

24 demonstrating that both reducing agents resulted in the same

25 number of protein identifications [12, 13]. Nevertheless,

26 acrylamide appeared to be more efficient in combination with

27 DTT, whereas iodoacetamide performed better with TCEP

28 [12]. As expected, alkylation reagent-related mass shifts were

29 the most abundant artefacts (e.g., +57.01 for IAA with 61582

30 and 53462 occurrences). However the frequently reported

31 methionine alkylation and the subsequent neutral loss of -48

32 Da from the molecular peptide ion $\left[\mathrm{M}-48+\mathrm{H}^{+}\right]^{+}$, which is the

33 loss of the side-chain from oxidised methionine (Mueller \&

34 Winter [12]), was rarely detected. Moreover, double alkylation

35 with the characteristic mass delta of +114 Da was compared to

36 counts for single alkylation ( $+57 \mathrm{Da}$ ) for all samples (on

37 average) below $<0.20 \%$. That mass addition was also observed

38 in the non-alkylated sample, suggesting that this mass shift

39 resulted rather from ubiquitinylation ('GlyGly') and not from

40 excessive alkylation. The modification analysis further

41 indicated that the applied alkylation procedure was sufficiently

42 mild (in particular when removing excess reagent before over

43 night digestion) with only little off-target, and hardly any

44 excessive, alkylation taking place. On the other hand, all

45 protocols that used IAA showed additional +39.98 Da mass

46 additions, which presumably derive from an N-terminal S-

47 carbamoylmethyl- cysteine cyclisation product [28, 71, 72].

48 Acrylamide showed only some additional -73.05 Da mass

49 shifts at low frequency, the mechanism of which, however

50 remains to be investigated. Interestingly, the protocol (10)

51 employing reduction but no alkylation resulted in an additional

52 series of (albeit low frequent) mass shifts (such as -59.05, 79.9,

$5394.9,99.0$ ), which likely originate from reactions of the free

54 sulfhydryl group with compounds (naturally) being present in
55 cellular extracts. An unexpected observation was the very abundant mass addition of +40.03 Da (labelled in Unimod http://www.unimod.org/ as 'propionaldehyde'). This mass shift was present in all protocols that employed protein precipitation with acetone, but was strongly reduced for protocols that used TCA with acetone wash, and which was absent in protocols using FASP. This mass shift was in a recent study related to acetone artefacts, which in fact can impact some $5 \%$ of the proteome (peptides with glycine as the second N-terminal amino acid) [17]. The 40 Da mass shift, however, can also be misinterpreted for an amino acid substitution from glycine to proline. Acetone modification involves aldimine formation between the ketone and the amino groups of a peptide, which is regarded as being acid labile and, therefore, rather rearranges to a more stable form (imidazolidinone), resulting in the observed +40.03 Da mass addition [17]. Interestingly, we observed some few mass additions of +41.03 $\mathrm{Da}$, which may indicate the presence of the proposed aldimine intermediate. Other artefacts that presumably also derive from acetone, such as $+98 \mathrm{Da}$ and to a lesser extent +84 and +112 Da [73], were only observed at trace levels or were not present at all. However, it is suggested that those artefacts seemed to be more pronounced at elevated $\mathrm{pH}$ and, therefore, our protocols might have prevented their formation. Even though acetone may increase peptide complexity substantially, this procedure is still commonly used in sample preparations, likely because it efficiently removes organic compounds such as inhibitors or compromising detergents, such as SDS [48]. The +28 Da mass adduct was nearly exclusively found for protocols in which formic acid was used during solid-phase extraction, confirming that yeast (at the given growth conditions), showed hardly any double methylation events. Previous studies provided similar findings, thereby highlighting that the proteome analysis outcome can be strongly affected by the use of this reagent $[25,26]$. A recent work demonstrated that these unwanted modifications can be largely avoided by processing the samples at low temperatures [25]. Another interesting observation in regard to solid-phase extraction was that the number of ammonium adductions (+17 Da) was hardly impacted by the nature of the buffers used to elute the peptides from the solid-phase extraction cartridges. Those adducts were supposedly carried over from the ammonium bicarbonate buffers, used for proteolytic digestion.

For the samples prepared with the alternative filter-aided protocols, a number of mass shifts were observed that were detected at a much lesser frequency in samples prepared by insolution digestion (e.g., one protocol: $+41.03,42.00$ or both protocols: $-89.05,11,0$ and -2,0). On the other hand (albeit at low frequency), a series of mass modifications such as -119.0 , $-72.0,11.98,12.0,42.0,125.07,128.07,156.09$ and 183.03 were increased or exclusively observed, when using filteraided protocols. 
1 Considerable variations in the fraction of overall 2 identified spectra observed for the different sample 3 preparation protocols. The number of identified MS/MS 4 spectra for each sample was determined by combining 5 identifications from TagGraph, PEAKS database search and 6 the sugar oxonium ion fragment search. First, the spectra 7 were de novo sequenced, where a fraction of all spectra (2.6 $8 \%$ and $3.8 \%$, on average, for replicates 1 and 2 , respectively) 9 did not provide any de novo spectra, which were further 10 allocated to the 'unsequenced' category ('no ALC score', 11 Figure 6, Supplemental Table S6). The de novo sequences 12 were then used to perform an unrestricted peptide 13 (modification) search using TagGraph, which resulted in the 14 identification of $28 \%$ and $26 \%$ of unmodified and $17 \%$ and $1514 \%$ of modified peptides, on average, for replicates 1 and 162 , respectively (at a 1\% peptide FDR). Moreover, PEAKS 17 was used as an additional/orthogonal search engine to 18 maximise identifications of unmodified peptides. Thereby, 19 the number of confidently identified peptides considerably 20 increased to $20 \%$ and $22 \%$, on average, for replicates 1 and 212 , respectively. Because different search engines employ 22 individual approaches and scoring matrices, a combination 23 of multiple search engines typically achieves significantly 24 more matches [5, 7, 74-79]. The PEAKS database 25 (PEAKS-DB) is a hybrid approach that combines elements 26 of de novo sequencing (short sequence tag extraction) and 27 database searching [80]. TagGraph, contrarily, is an 28 unrestricted de novo sequence-tag approach employing fast 29 string-based searches followed by a probabilistic validation 30 model optimised for the identification of modified peptides 31 [41]. Furthermore, spectra were screened for known 32 carbohydrate fragments, which indicate a glycopeptide 33 spectrum that would likely remain unidentified by any of 34 the above-employed approaches and, therefore, were 35 allocated to the category 'sugars' $(0.8 \%$ and $0.6 \%$, on 36 average, for replicates 1 and 2, respectively). Finally, the 37 remainder of the de novo sequences that could not be 38 allocated to one of the aforementioned categories were 39 categorised as 'unidentified'. Here, approximately 31 and $4034 \%$ remained unidentified for replicates 1 and 2 , 41 respectively.

42 As mentioned above, small differences in the identification 43 rates between the two biological replicates were observed, 44 presumably due to operational performance differences of 45 the mass spectrometer at the different time periods. Still, a 46 very similar pattern in allocation of the sequencing spectra 47 is observed between the replicates (Figure 6). Expectedly, 48 protocols 6 (methanol quenching), 8 (TCA precipitation) 49 and 14 (FASP with SDS buffer) resulted in the lowest 50 number of spectral identifications due to possible protein 51 aggregation and following losses of protein during precipitation and LC-MS/MS incompatibility of reagents, respectively. Furthermore, the number of additional identifications with PEAKS-DB was considerably lower than for the other protocols when the samples were quenched with methanol. Conversely, a higher number of spectra from potential glycopeptides was observed using this protocol. This observation might be explained by the slightly increased fraction of membrane proteins (Figure 3), when using this protocol (number 6). The other protocols resulted in highly similar profiles and identification rates, with few exceptions. A considerably lower number of modified peptides was found for protocols 10 (no alkylation) and 13 (FASP, Urea). As speculated in the previous paragraph, this presumably results from the loss of cysteine-containing peptides, and acetone induced modifications for protocols 10 and 13. Finally, sample 13 (FASP with Urea buffer) resulted in one of the highest identification rates with a relatively low number of modified peptides. Nevertheless, a large fraction of all spectra remained unidentified.

Investigating 'the nature of the unidentified fraction' As also observed in other studies [1,2], approximately one third of the MS/MS spectra remained unidentified in the current study across all protocols. Recurrently, protocols 6 , 8 and 14 led to a significantly higher fraction of unidentified spectra, on average, $44 \%, 54 \%$ and $45 \%$, respectively. Other protocols obtained similar results, ranging from 30 $35 \%$ unidentified scans. Protocol 7 (in-solution, IAA) resulted in the highest number of identified spectra $(70 \%)$, showcasing the key role of the type of protocol used on the identification rate. The inability to identify spectra can have various causes, such as impurities (or contaminations) in the sample, excessive co-fragmentation, poor quality spectra, unexpected modifications or peptide sequences not covered by the database (e.g., sequence variants and microbial impurities). First, the taxonomic purity of the samples was confirmed by a recently published de novo metaproteomic profiling approach [40], in order to exclude the presence of sample carry-over from foregoing analyses and to exclude the presence of bacterial contaminants. This was performed with protocol 13 for both biological replicates, for which no impurities could be identified (data not shown), confirming that no carry-over took place and that the yeast cultures were of highest purity.

A high quality of the fragmentation spectra is a prerequisite for confident peptide spectrum matching. Therefore, we assessed the quality by de novo sequencing and the associated average local confidence score (ALC), as determined by PEAKS [39]. In general, peptides with higher ALC scores indicate a more complete peptide 
1 fragment ion coverage and, therefore, are expected to have

2 a higher chance of correct identification. As example, the

3 ALC scores of the (un)identified spectra of biological

4 replicate 1 processed with protocol 13 is visualised in

5 Figure 7 (Supplemental Table S6). The ALC distribution is,

6 as expected, very different for the fraction of identified and

7 unidentified spectra. The ALC scores of the unidentified

8 spectra spread predominantly around low numbers (where

9 all identified spectra show very high ALC scores),

10 suggesting that reduced quality (peptide fragment ion

11 coverage) is the main reason for the lack of identification.

12 Nevertheless, a small fraction of the unidentified spectra

13 showed high ALC scores. Most of the de novo sequences

14 were close to sequences in the yeast protein sequence

15 database and may therefore represent sequence variants

16 (e.g., single nucleotide polymorphisms SNPs, or isoform-

17 and allele-specific variants of proteins) or additional peptide

18 modifications, which were not covered by the database or

19 could not even be identified by the open modification search

20 approach. Furthermore, peptides containing unexpected

21 semi-tryptic or nonspecific cleavages may also add to this

22 fraction [21].

\section{CONCLUSION}

Our systematic study on the unicellular organism yeast demonstrates the strong impact of several elements within sample preparation protocols, on various aspects of the proteomics analysis outcome. The total number of obtained peptide spectrum matches ranged from approx. $1 \times 10^{4}$ to $3 \times 10^{4}$, the proteome coverage ranged from below $15 \%$ to approx. $30 \%$, and the overall matched spectra varied in the range from approx. 50 to $70 \%$. The depth of the proteome coverage was heavily affected by the sample quenching protocol, where cells arrested by TCA resulted in a higher number of protein identifications compared to methanol-arrested cells. Furthermore, filter-aided procedures, when combined with Urea, outperformed other protocols in terms of the number of protein identifications.

The use of unrestricted modification search moreover enabled to examine for reagent-induced peptide modifications. The overall frequency of reagent-specific modifications was for some protocols significantly lower (particularly for protocols 8 and 14), albeit that also correlated with the lower number of spectra and decreased total ion current (TIC) observed for those protocols. Our analysis confirmed previously identified sample processing induced modifications, but also revealed unexpected modifications, such as those related to acetone, alkylating agents as well as filter materials.

Approximately $70 \%$ of the overall acquired MS/MS spectra could be identified when using filter-aided as well as the best performing in-solution protocols. The unidentified spectra were predominantly of low-quality, lacking

\section{Author Contributions}

81 MP, PDL and MDR designed the experiments. MDR, EK 82 and WB performed experiments. MDR and MP analyzed 83 the data. MDR and MP wrote the manuscript. All authors

sufficient peptide fragment coverage for a confident identification. A maximum proteome coverage and reduced number of reagent-induced modifications were obtained for the filter-aided approach, even in the presence of Urea buffer (when using moderate incubation temperatures). However, this approach appeared to be more laborious and requires the availability of suitable filters and centrifuges. A comparable performance was obtained with the best performing in-solution protocols, such as 7 and 11. More specialised protocols, such as S-trap [81] and ultrasonic FASP digestion [82] were not evaluated, but are expected to enable a similarly good proteome coverage at reduced processing times.

Ultimately, we systematically evaluated a matrix of sample preparation protocols for the unicellular model eukaryote yeast, and provide a large resource of protocols and associated mass spectrometric raw data. These enable the selection of suitable sample preparation elements, the design of more specialised procedures and support the evaluation and analysis of (native) peptide modifications in yeast.

\section{AUTHOR INFORMATION}

*Corresponding Author: Delft University of Technology, Department of Biotechnology, van der Maasweg 9, 2629 HZ Delft, The Netherlands. E-mail: m.pabst@tudelft.nl. have given approval to the final version of the manuscript.

\section{Notes}

This work was supported by a TU Delft startup fund. Conflict of interest-The authors declare no competing interests.

\section{ACKNOWLEDGMENT}

The authors are grateful to valuable discussions with their col- leagues from the department of Biotechnology and acknowledge Carol de Ram for technical support.

\section{REFERENCES}

1. Chick, J.M., Kolippakkam, D., Nusinow, D.P., Zhai, B., Rad, R., Huttlin, E.L., Gygi, S.P.: A mass-tolerant database search identifies a large proportion of unassigned spectra in shotgun proteomics as modified peptides. 33, (2015). https://doi.org/10.1038/nbt.3267

2. $\quad$ Griss, J., Perez-Riverol, Y., Lewis, S., Tabb, D.L., Dianes, J.A., Del-Toro, N., Rurik, M., Walzer, M., Kohlbacher, O., Hermjakob, H., Wang, R., Vizcano, J.A.: Recognizing millions of consistently unidentified 
bioRxiv preprint doi: https://doi.org/10.1101/2022.01.17.476533; this version posted January 20, 2022. The copyright holder for this preprint (which was not certified by peer review) is the author/funder. All rights reserved. No reuse allowed without permission.

Maxime den Ridder et al., 2022

1 spectra across hundreds of shotgun proteomics datasets. Nat. Methods. 13, 2 651-656 (2016). https://doi.org/10.1038/nmeth.3902

$33 . \quad$ Ning, K., Fermin, D., Nesvizhskii, A.I.: Computational analysis 4 of unassigned high-quality MS/MS spectra in proteomic data sets. 5 Proteomics. $10, \quad 2712-2718$ (2010). 6 https://doi.org/10.1002/pmic.200900473

$74 . \quad$ Nesvizhskii, A.I., Roos, F.F., Grossmann, J., Vogelzang, M.,

8 Eddes, J.S., Gruissem, W., Baginsky, S., Aebersold, R.: Dynamic spectrum 9 quality assessment and iterative computational analysis of shotgun modifications, sequence polymorphisms, and novel peptides. Mol. Cell. Proteomics. 5, 652-670 (2006). https://doi.org/10.1074/mcp.M500319MCP200

5. $\quad$ Tu, C., Sheng, Q., Li, J., Ma, D., Shen, X., Wang, X., Shyr, Y., Yi, Z., Qu, J.: Optimization of search engines and postprocessing approaches to maximize peptide and protein identification for highresolution mass data. J. Proteome Res. 14, 4662-4673 (2015). https://doi.org/10.1021/acs.jproteome.5b00536

6. $\quad$ Shteynberg, D., Nesvizhskii, A.I., Moritz, R.L., Deutsch, E.W.: Combining results of multiple search engines in proteomics. Mol. Cell. Proteomics.

$$
\text { 12, 2383-2393 }
$$

(2013). https://doi.org/10.1074/mcp.R113.027797

$7 . \quad$ Yu, W., Taylor, J.A., Davis, M.T., Bonilla, L.E., Lee, K.A., Auger, P.L., Farnsworth, C.C., Welcher, A.A., Patterson, S.D.: Maximizing the sensitivity and reliability of peptide identification in largescale proteomic experiments by harnessing multiple search engines. Proteomics. 10 1172-1189

(2010). https://doi.org/10.1002/pmic.200900074

8. Piehowski, P.D., Petyuk, V.A., Orton, D.J., Xie, F., Moore, R.J., Ramirez-Restrepo, M., Engel, A., Lieberman, A.P., Albin, R.L., Camp, D.G., Smith, R.D., Myers, A.J.: Sources of technical variability in quantitative LC-MS proteomics: Human brain tissue sample analysis. J. Proteome Res. 12, 2128-2137 (2013). https://doi.org/10.1021/pr301146m 9. Carapito, C., Klemm, C., Aebersold, R., Domon, B.: Systematic LC-MS Analysis of Labile Post-Translational Modifications in Complex Mixtures. 2608-2614 (2009)

10. Grassl, J., Westbrook, J.A., Robinson, A., Borén, M., Dunn, M.J., Clyne, R.K.: Preserving the yeast proteome from sample degradation. Proteomics. 9 $4616-4626$

(2009). https://oi.org/10.1002/pmic.200800945

11. Kanshin, E., Tyers, M., Thibault, P.: Sample Collection Method Bias Effects in Quantitative Phosphoproteomics. J. Proteome Res. 14, 2998-3004 (2015). https://doi.org/10.1021/acs.jproteome.5b00404 12. Müller, T., Winter, D.: Systematic Evaluation of Protein Reduction and Alkylation Reveals Massive Unspecific Side Effects by lodine-containing Reagents. Mol. Cell. Proteomics. 16, 1173-1187 (2017). https://doi.org/10.1074/mcp.m116.064048

13. Suttapitugsakul, S., Xiao, H., Smeekens, J., Wu, R.: Evaluation and optimization of reduction and alkylation methods to maximize peptide dentification with MS-based proteomics. Mol. Biosyst. 13, 2574-2582 2017). https://doi.org/10.1039/c7mb00393e

14. Güray, M.Z., Zheng, S., Doucette, A.A., Niu, L., Zhang, H., Wu, Z., Wang, Y., Liu, H., Wu, X., Wang, W., Simpson, D.M., Beynon, R.J., Jiang, L., He, L., Fountoulakis, M.: Mass Spectrometry of Intact Proteins Reveals +98 u Chemical Artifacts Following Precipitation in Acetone. J. Proteome Res. 16, 444-450 (2018). https://doi.org/10.1021/acs.jproteome.6b00841

15. Santa, C., Anjo, S.I., Manadas, B.: Protein precipitation of diluted samples in SDS-containing buffer with acetone leads to higher protein recovery and reproducibility in comparison with TCA/acetone approach. Proteomics. 16, 1847-1851 (2016). https://doi.org/10.1002/pmic.201600024

16. Jiang, L., He, L., Fountoulakis, M.: Comparison of protein precipitation methods for sample preparation prior to proteomic analysis. $\begin{array}{lllll}\text { J. Chromatogr. A. } & \text { A023, } 317-320 \quad \text { (2004). }\end{array}$ https://doi.org/10.1016/j.chroma.2003.10.029

7. Simpson, D.M., Beynon, R.J.: Acetone precipitation of proteins and the modification of peptides. J. Proteome Res. 9, 444-450 (2010). https://doi.org/10.1021/pr900806x
7018.
18. Crowell, A.M.J., Wall, M.J., Doucette, A.A.: Maximizing recovery of water-soluble proteins through acetone precipitation. Anal Chim. Acta. 796, 48-54 (2013). https://doi.org/10.1016/j.aca.2013.08.005 19. Wiśniewski, J.R., Zougman, A., Nagaraj, N., Mann, M.: Universal sample preparation method for proteome analysis. Nat. Methods. 6, 359-362 (2009). https://doi.org/10.1038/nmeth.1322

20. Manza, L.L., Stamer, S.L., Ham, A.J.L., Codreanu, S.G., Liebler, D.C.: Sample preparation and digestion for proteomic analyses

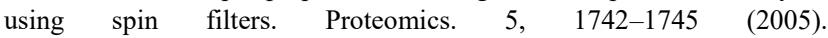
https://doi.org/10.1002/pmic.200401063

21. Lin, Z., Ren, Y., Shi, Z., Zhang, K., Yang, H., Liu, S., Hao, P.: Evaluation and minimization of nonspecific tryptic cleavages in proteomic sample preparation. Rapid Commun. Mass Spectrom. 34, (2020). https://doi.org/10.1002/rcm.8733

22. Cañas, B., Piñeiro, C., Calvo, E., López-Ferrer, D., Gallardo, J.M.: Trends in sample preparation for classical and second generation proteomics. J. Chromatogr. A. 1153, 235-258 (2007). https://doi.org/10.1016/j.chroma.2007.01.045

23. Klont, F., Bras, L., Wolters, J.C., Ongay, S., Bischoff, R., Halmos, G.B., Horvatovich, P.: Assessment of Sample Preparation Bias in Mass Spectrometry-Based Proteomics. Anal. Chem. 90, 5405-5413 (2018). https://doi.org/10.1021/acs.analchem.8b00600

24. Jiang, T., Zhou, X., Taghizadeh, K., Dong, M., Dedon, P.C.: Nformylation of lysine in histone proteins as a secondary modification arising from oxidative DNA damage. Proc. Natl. Acad. Sci. 104, 60-65 (2007). https://doi.org/10.1073/pnas.0606775103

25. Lenčo, J., Khalikova, M.A., Švec, F.: Dissolving Peptides in $0.1 \%$ Formic Acid Brings Risk of Artificial Formylation. J. Proteome Res. 19, 993-999 (2020). https://doi.org/10.1021/acs.jproteome.9b00823

26. Zheng, S., Doucette, A.A.: Preventing N- and O-formylation of proteins when incubated in concentrated formic acid. Proteomics. 16, 1059-1068 (2016). https://doi.org/10.1002/pmic.201500366

27. Kollipara, L., Zahedi, R.P.: Protein carbamylation: In vivo modification or in vitro artefact? Proteomics. 13, 941-944 (2013). https://doi.org/10.1002/pmic.201200452

28. Boja, E.S., Fales, H.M.: Overalkylation of a protein digest with iodoacetamide. Anal. Chem. 73, 3576-3582 (2001). https://doi.org/10.1021/ac0103423

29. Kuznetsova, K.G., Levitsky, L.I., Pyatnitskiy, M.A., Ilina, I.Y., Bubis, J.A., Solovyeva, E.M., Zgoda, V.G., Gorshkov, M. V., Moshkovskii, S.A.: Cysteine alkylation methods in shotgun proteomics and their possible effects on methionine residues. J. Proteomics. 231, 104022 (2021). https://doi.org/10.1016/j.jprot.2020.104022

$30 . \quad$ Wiśniewski, J.R., Zettl, K., Pilch, M., Rysiewicz, B., Sadok, I.: 'Shotgun' proteomic analyses without alkylation of cysteine. Anal. Chim. Acta. 1100, 131-137 (2020). https://doi.org/10.1016/j.aca.2019.12.007 31. Liu, S., Zhang, C., Campbell, J.L., Zhang, H., Yeung, K.K., Han, V.K.M., Lajoie, G.A.: Formation of phosphopeptide-metal ion complexes in liquid chromatography / electrospray mass spectrometry and their influence on phosphopeptide detection. 2747-2756 (2005) https://doi.org/10.1002/rcm.2105

32. Hao, P., Ren, Y., Alpert, A.J., Sze, S.K.: Detection, Evaluation and Minimization of Nonenzymatic Deamidation in Proteomic Sample Preparation. Mol. Cell. Proteomics. 10, O111.009381 (2011). https://doi.org/10.1074/mcp.o111.009381

33. Purwaha, P., Silva, L.P., Hawke, D.H., Weinstein, J.N., Lorenzi, P.L.: An artifact in LC-MS/MS measurement of glutamine and glutamic acid: In-source cyclization to pyroglutamic acid. Anal. Chem. 86, 5633-5637 (2014). https://doi.org/10.1021/ac501451v

34. Stadtman, E.R., Van Remmen, H., Richardson, A., Wehr, N.B., Levine, R.L.: Methionine oxidation and aging. Biochim. Biophys. Acta Proteins Proteomics. 1703, 135-140 (2005). https://doi.org/10.1016/j.bbapap.2004.08.010

35. Entian, K.-D., Kötter, P.: 25 Yeast Genetic Strain and Plasmid Collections. Methods Microbiol. 36, 629-666 (2007). https://doi.org/10.1016/S0580-9517(06)36025-4

36. Solis-Escalante, D., Kuijpers, N.G.A., Barrajon-Simancas, N., van den Broek, M., Pronk, J.T., Daran, J.M., Daran-Lapujade, P.: A Minimal set of glycolytic genes reveals strong redundancies in 
bioRxiv preprint doi: https://doi.org/10.1101/2022.01.17.476533; this version posted January 20, 2022. The copyright holder for this preprint (which was not certified by peer review) is the author/funder. All rights reserved. No reuse allowed without permission.

Maxime den Ridder et al., 2022

1 saccharomyces cerevisiae central metabolism. Eukaryot. Cell. 14, 804-816 2 (2015). https://doi.org/10.1128/EC.00064-15

$337 . \quad$ Verduyn, C., Postma, E., Scheffers, W.A., Van Dijken, J.P.: 4 Effect of benzoic acid on metabolic fluxes in yeasts: A continuous-culture 5 study on the regulation of respiration and alcoholic fermentation. Yeast. 8, 6 501-517 (1992). https://doi.org/10.1002/yea.320080703

$738 . \quad$ Köcher, T., Pichler, P., Swart, R., Mechtler, K.: Analysis of 8 protein mixtures from whole-cell extracts by single-run nanolc-ms $/ \mathrm{ms}$ 9 using ultralong gradients. Nat. Protoc. 7, 882-890 (2012). 10 https://doi.org/10.1038/nprot.2012.036

$1139 . \quad$ Ma, B., Zhang, K., Hendrie, C., Liang, C., Li, M., Doherty12 Kirby, A., Lajoie, G.: PEAKS: Powerful software for peptide de novo 13 sequencing by tandem mass spectrometry. Rapid Commun. Mass 14 Spectrom. 17, 2337-2342 (2003). https://doi.org/10.1002/rcm.1196

15 40. Kleikamp, H.B.C., Pronk, M., Tugui, C., Guedes da Silva, L.,

16 Abbas, B., Lin, Y.M., van Loosdrecht, M.C.M., Pabst, M.: Database-

17 independent de novo metaproteomics of complex microbial communities.

18 Cell Syst. $\quad 12, \quad 375-383 . e 5 \quad$ (2021).

19 https://doi.org/10.1016/j.cels.2021.04.003

20 41. Devabhaktuni, A., Lin, S., Zhang, L., Swaminathan, K., 21 Gonzalez, C.G., Olsson, N., Pearlman, S.M., Rawson, K., Elias, J.E.: 46. Carbon, S., Douglass, E., Good, B.M., Unni, D.R., Harris, N.L., Mungall, C.J., Basu, S., Chisholm, R.L., Dodson, R.J., Hartline, E., Fey, P., Thomas, P.D., Albou, L.P., Ebert, D., Kesling, M.J., Mi, H., D.A., Antonazzo, G., Attrill, H., Brown, N.H., Garapati, P., Marygold, S.J., Trovisco, V., dos Santos, G., Falls, K., Tabone, C., Zhou, P., Goodman, J.L., Strelets, V.B., Thurmond, J., Garmiri, P., Ishtiaq, R., Rodríguez62 Thurlow, K.E., Chibucos, M., Giglio, M., Nadendla, S., Munro, J., 62 Jackson, R., Duesbury, M.J., Del-Toro, N., Meldal, B.H.M., Paneerselvam, 63 K., Perfetto, L., Porras, P., Orchard, S., Shrivastava, A., Chang, H.Y., Finn, 64 R.D., Mitchell, A.L., Rawlings, N.D., Richardson, L., Sangrador-Vegas, 65 A., Blake, J.A., Christie, K.R., Dolan, M.E., Drabkin, H.J., Hill, D.P., Ni, 66 L., Sitnikov, D.M., Harris, M.A., Oliver, S.G., Rutherford, K., Wood, V., 67 Hayles, J., Bähler, J., Bolton, E.R., de Pons, J.L., Dwinell, M.R., Hayman, 68 G.T., Kaldunski, M.L., Kwitek, A.E., Laulederkind, S.J.F., Plasterer, C., 69
J.P., Aleksander, S.A., Alexander, M.J., Cherry, J.M., Engel, S.R., Gondwe, F., Karra, K., Miyasato, S.R., Nash, R.S., Simison, M., Skrzypek, M.S., Weng, S., Wong, E.D., Feuermann, M., Gaudet, P., Morgat, A., Bakker, E., Berardini, T.Z., Reiser, L., Subramaniam, S., Huala, E., Arighi, C.N., Auchincloss, A., Axelsen, K., Argoud-Puy, G., Bateman, A., Blatter, M.C., Boutet, E., Bowler, E., Breuza, L., Bridge, A., Britto, R., Bye-A-Jee, H., Casas, C.C., Coudert, E., Denny, P., Es-Treicher, A., Famiglietti, M.L., Georghiou, G., Gos, A.N., Gruaz-Gumowski, N., Hatton-Ellis, E., Hulo, C., Ignatchenko, A., Jungo, F., Laiho, K., Le Mercier, P., Lieberherr, D. Lock, A., Lussi, Y., MacDougall, A., Ma-Grane, M., Martin, M.J., Masson, P., Natale, D.A., Hyka-Nouspikel, N., Orchard, S., Pedruzzi, I., Pourcel, L., Poux, S., Pundir, S., Rivoire, C., Speretta, E., Sundaram, S., Tyagi, N., Warner, K., Zaru, R., Wu, C.H., Diehl, A.D., Chan, J.N., Grove, C., Lee, R.Y.N., Muller, H.M., Raciti, D., van Auken, K., Sternberg, P.W., Berriman, M., Paulini, M., Howe, K., Gao, S., Wright, A., Stein, L., Howe, D.G., Toro, S., Westerfield, M., Jaiswal, P., Cooper, L., Elser, J.: The Gene Ontology resource: Enriching a GOld mine. Nucleic Acids Res. 49, D325D334 (2021). https://doi.org/10.1093/nar/gkaa1113

47. Bateman, A.: UniProt: A worldwide hub of protein knowledge. Nucleic Acids Res. 47, D506-D515 (2019). https://doi.org/10.1093/nar/gky1049

48. Nickerson, J.L., Doucette, A.A.: Rapid and Quantitative Protein Precipitation for Proteome Analysis by Mass Spectrometry. J. Proteome Res. 19, 2035-2042 (2020). https://doi.org/10.1021/acs.jproteome.9b00867

49. Pérez-Rodriguez, S., Ramírez, O.T., Trujillo-Roldán, M.A., Valdez-Cruz, N.A.: Comparison of protein precipitation methods for sample preparation prior to proteomic analysis of Chinese hamster ovary cell homogenates. Electron. J. Biotechnol. 48, 86-94 (2020). https://doi.org/10.1016/j.ejbt.2020.09.006

50. Rajalingam, D., Loftis, C., Xu, J.J., Kumar, T.K.S.: Trichloroacetic acid-induced protein precipitation involves the reversible association of a stable partially structured intermediate. Protein Sci. 18, 980-993 (2009). https://doi.org/10.1002/pro.108

51. Manadas, B.J., Vougas, K., Fountoulakis, M., Duarte, C.B. Sample sonication after trichloroacetic acid precipitation increases protein recovery from cultured hippocampal neurons, and improves resolution and reproducibility in two-dimensional gel electrophoresis. Electrophoresis. 27, 1825-1831 (2006). https://doi.org/10.1002/elps.200500757

52. Lin, Y., Zhou, J., Bi, D., Chen, P., Wang, X., Liang, S.: Sodiumdeoxycholate-assisted tryptic digestion and identification of proteolytically resistant proteins. Anal. Biochem. 377, 259-266 (2008). https://doi.org/10.1016/j.ab.2008.03.009

53. Hustoft, H.K., Reubsaet, L., Greibrokk, T., Lundanes, E., Malerod, H.: Critical assessment of accelerating trypsination methods. J. Pharm. Biomed. Anal. 56, 1069-1078 (2011). https://doi.org/10.1016/j.jpba.2011.08.013

54. Scheerlinck, E., Dhaenens, M., Van Soom, A., Peelman, L., De Sutter, P., Van Steendam, K., Deforce, D.: Minimizing technical variation during sample preparation prior to label-free quantitative mass spectrometry. Anal. Biochem. 490, 14-19 (2015). https://doi.org/10.1016/j.ab.2015.08.018

55. Canelas, A.B., Ras, C., ten Pierick, A., van Dam, J.C., Heijnen, J.J., van Gulik, W.M.: Leakage-free rapid quenching technique for yeast metabolomics. Metabolomics. 4, 226-239 (2008). https://doi.org/10.1007/s11306-008-0116-4

56. Schmidt, S.A., Jacob, S.S., Ahn, S.B., Rupasinghe, T., Krömer, J.O., Khan, A., Varela, C.: Two strings to the systems biology bow: Coextracting the metabolome and proteome of yeast. Metabolomics. 9, 173188 (2013). https://doi.org/10.1007/s11306-012-0437-1

57. Ludwig, K.R., Schroll, M.M., Hummon, A.B.: Comparison of In-Solution, FASP, and S-Trap Based Digestion Methods for Bottom-Up Proteomic Studies. J. Proteome Res. 17, 2480-2490 (2018). https://doi.org/10.1021/acs.jproteome.8b00235

58. Tanca, A., Biosa, G., Pagnozzi, D., Addis, M.F., Uzzau, S.: Comparison of detergent-based sample preparation workflows for LTQOrbitrap analysis of the Escherichia coli proteome. Proteomics. 13, 25972607 (2013). https://doi.org/10.1002/pmic.201200478 
bioRxiv preprint doi: https://doi.org/10.1101/2022.01.17.476533; this version posted January 20, 2022. The copyright holder for this

preprint (which was not certified by peer review) is the author/funder. All rights reserved. No reuse allowed without permission.

Maxime den Ridder et al., 2022

159

9 Poughlin, E.E., Westphall, M.S., Coon, J.J.: The One Hour Yeast

9 Proteome. Mol. Cell. Proteomics. 13, 339-347 (2014).

10 https://doi.org/10.1074/mcp.M113.034769

11 62. Thakur, S.S., Geiger, T., Chatterjee, B., Bandilla, P., Froḧlich,

12 F., Cox, J., Mann, M.: Deep and highly sensitive proteome coverage by

13 LC-MS/MS without prefractionation. Mol. Cell. Proteomics. 10, 1-9

14 (2011). https://doi.org/10.1074/mcp.M110.003699

15 63. Gao, Y., Ping, L., Duong, D., Zhang, C., Dammer, E.B., Li, Y.,

16 Chen, P., Chang, L., Gao, H., Wu, J., Xu, P.: Mass-Spectrometry-Based

17 Near-Complete Draft of the Saccharomyces cerevisiae Proteome. J.

18 Proteome Res. 20, 1328-1340 (2021).

19 https://doi.org/10.1021/acs.jproteome.0c00721

2064 Li, X., Gerber, S.A., Rudner, A.D., Beausoleil, S.A., Haas, W., 21 Villén, J., Elias, J.E., Gygi, S.P.: Large-scale phosphorylation analysis f? -factor-arrested Saccharomyces cerevisiae. J. Proteome Res. 6, 1190197 (2007). https://doi.org/10.1021/pr060559j

65. Han, G., Ye, M., Zhou, H., Jiang, X., Feng, S., Jiang, X., Tian, R., Wan, D., Zou, H., Gu, J.: Large-scale phosphoproteome analysis of human liver tissue by enrichment and fractionation of phosphopeptides with strong anion exchange chromatography. Proteomics. 8, 1346-1361 (2008). https://doi.org/10.1002/pmic.200700884

66. Swaney, D.L., Wenger, C.D., Coon, J.J.: Value of Using Multiple Proteases for Large-Scale Mass Spectrometry-Based Proteomics research articles. 1323-1329 (2010)

67. Giansanti, P., Tsiatsiani, L., Low, T.Y., Heck, A.J.R.: Six alternative proteases for mass spectrometry-based proteomics beyond trypsin. Nat. Protoc. 11, 993-1006 (2016). https://doi.org/10.1038/nprot.2016.057

68. Daran-Lapujade, P., Jansen, M.L.A., Daran, J.M., Van Gulik, W., De Winde, J.H., Pronk, J.T.: Role of Transcriptional Regulation in Controlling Fluxes in Central Carbon Metabolism of Saccharomyces cerevisiae: A chemostat culture study. J. Biol. Chem. 279, 9125-9138 (2004). https://doi.org/10.1074/jbc.M309578200

69. Kollipara, L., Zahedi, R.P.: Protein carbamylation: In vivo modification or in vitro artefact? Proteomics. 13, 941-944 (2013). https://doi.org/10.1002/pmic.201200452

70. Sun, S., Zhou, J.Y., Yang, W., Zhang, H.: Inhibition of protein carbamylation in Urea solution using ammonium-containing buffers. Anal. Biochem. 446, 76-81 (2014). https://doi.org/10.1016/j.ab.2013.10.024 71. Tarasova, I.A., Chumakov, P.M., Moshkovskii, S.A., Gorshkov, M. V.: Profiling modifications for glioblastoma proteome using ultra-tolerant database search: Are the peptide mass shifts biologically elevant or chemically induced? J. Proteomics. 191, 16-21 (2019). https://doi.org/10.1016/j.jprot.2018.05.010

2. Geoghegan, K.F., Hoth, L.R., Tan, D.H., Borzilleri, K.A., Withka, J.M., Boyd, J.G.: Cyclization of N-terminal Scarbamoylmethylcysteine causing loss of $17 \mathrm{Da}$ from peptides and extra peaks in peptide maps. J. Proteome Res. 1, 181-187 (2002). https://doi.org/10.1021/pr025503d

73. Güray, M.Z., Zheng, S., Doucette, A.A.: Mass Spectrometry of Intact Proteins Reveals +98 u Chemical Artifacts Following Precipitation

6174 ://doi.org/10.1021/acs.jproteome.6b00841

Shteynberg, D., Nesvizhskii, A.I., Moritz, R.L., Deutsch, E.W .,

63 J., Editor, S., Walker, J.M., Yu, W., Taylor, J.A., Davis, M.T., Bonilla,

64 L.E., Lee, K.A., Auger, P.L., Farnsworth, C.C., Welcher, A.A., Patterson,

65 S.D., Searle, B.C., Turner, M., Nesvizhskii, A.I., Dagda, R.K., Sultana, T., 66 Lyons-Weiler, J., Alves, G., Wu, W.W., Wang, G., Shen, R.F., Yu, Y.K., 67 Kwon, T., Choi, H., Vogel, C., Nesvizhskii, A.I., Marcotte, E.M., Agten, 68 A., Van Houtven, J., Askenazi, M., Burzykowski, T., Laukens, K., 69 Valkenborg, D.: Visualizing the agreement of peptide assignments
70 between different search engines. J. Proteome Res. 7, 39-47 (2008).

71 https://doi.org/10.1002/jms.4471

72 75. Searle, B.C., Turner, M., Nesvizhskii, A.I.: Improving 73 sensitivity by probabilistically combining results from multiple MS/MS 74 search methodologies. J. Proteome Res. 7, 245-253 (2008) 75 https://doi.org/10.1021/pr070540w

$7676 . \quad$ Kwon, T., Choi, H., Vogel, C., Nesvizhskii, A.I., Marcotte, 77 E.M.: MSblender: A probabilistic approach for integrating peptide 78 identifications from multiple database search engines. J. Proteome Res. 10 ,

79 2949-2958 (2011). https://doi.org/10.1021/pr2002116

$8077 . \quad$ Alves, G., Wu, W.W., Wang, G., Shen, R.F., Yu, Y.K. 81 Enhancing peptide identification confidence by combining search 82 methods. J. Proteome Res. 7, 3102-3113 (2008). 83 https://doi.org/10.1021/pr700798h

$8478 . \quad$ Agten, A., Van Houtven, J., Askenazi, M., Burzykowski, T., 85 Laukens, K., Valkenborg, D.: Visualizing the agreement of peptide 86 assignments between different search engines. J. Mass Spectrom. 55, 87 (2020). https://doi.org/10.1002/jms.4471

$8879 . \quad$ Dagda, R.K., Sultana, T., Lyons-Weiler, J.: Evaluation of the 89 consensus of four peptide identification algorithms for Tandem mass 90 spectrometry based proteomics. J. Proteomics Bioinforma. 3, 39-47 91 (2010). https://doi.org/10.4172/jpb.1000119

$9280 . \quad$ Zhang, J., Xin, L., Shan, B., Chen, W., Xie, M., Yuen, D.,

93 Zhang, W., Zhang, Z., Lajoie, G.A., Ma, B.: PEAKS DB: De novo 94 sequencing assisted database search for sensitive and accurate peptide 95 identification. Mol. Cell. Proteomics. 11, 1-8 (2012). 96 https://doi.org/10.1074/mcp.M111.010587

97 81. Hailemariam, M., Eguez, R.V., Singh, H., Bekele, S., Ameni, 98 G., Pieper, R., Yu, Y.: S-Trap, an Ultrafast Sample-Preparation Approach 99 for Shotgun Proteomics. J. Proteome Res. 17, 2917-2924 (2018). 100 https://doi.org/10.1021/acs.jproteome.8b00505

101 82. Carvalho, L.B., Capelo-Martínez, J.L., Lodeiro, C., 102 Wiśniewski, J.R., Santos, H.M.: Ultrasonic-Based Filter Aided Sample 103 Preparation as the General Method to Sample Preparation in Proteomics. 104 Anal. Chem. 92, 9164-9171 (2020). 105 https://doi.org/10.1021/acs.analchem.0c01470 
Table 1: Matrix of investigated sample preparation protocols (1-14). Abbreviations: SPE, solid phase extraction; TCA, trichloroacetic acid; SDS, sodium dodecyl sulphate; DTT, Dithiothreitol; TCEP, Tris(2-carboxyethyl)phosphine; IAA, iodoacetamide; AA, acrylamide; TFA, trifluoroacetic acid; FA, formic acid; ABC, ammonium bicarbonate. The detailed protocols are provided in the S.I. materials.

\begin{tabular}{|c|c|c|c|c|c|c|}
\hline \# & $\begin{array}{l}\text { SAMPLING } \\
\text { METHOD }\end{array}$ & $\begin{array}{l}\text { LYSIS } \\
\text { BUFFER }\end{array}$ & $\begin{array}{l}\text { REDUCING } \\
\text { AGENT }\end{array}$ & $\begin{array}{l}\text { ALKYLATION } \\
\text { REAGENT }\end{array}$ & $\begin{array}{c}\text { PROTEIN } \\
\text { PURIFICATION }\end{array}$ & SPE BUFFERS \\
\hline 1 & TCA & SDS & DTT & IAA & Acetone & TFA \\
\hline 2 & TCA & SDS & DTT & IAA & Acetone & $F A+A B C$ \\
\hline 3 & TCA & SDS & DTT & IAA & Acetone & ABC \\
\hline 4 & TCA & SDS & DTT & IAA & Acetone & $\mathrm{MeOH}$ \\
\hline 5 & TCA & SDS & DTT & IAA & Acetone & FA \\
\hline 6 & $\mathrm{MeOH}$ & SDS & DTT & IAA & Acetone & $T F A+A B C$ \\
\hline 7 & TCA & SDS & DTT & IAA & Acetone & $T F A+A B C$ \\
\hline 8 & TCA & SDS & DTT & IAA & TCA & $\mathrm{TFA}+\mathrm{ABC}$ \\
\hline 9 & TCA & SDS & DTT & AA & Acetone & $T F A+A B C$ \\
\hline 10 & TCA & SDS & DTT & - & Acetone & $T F A+A B C$ \\
\hline 11 & TCA & SDS & TCEP & IAA & Acetone & $T F A+A B C$ \\
\hline 12 & TCA & UREA & DTT & IAA & Acetone & $T F A+A B C$ \\
\hline 13 & TCA & UREA & DTT & IAA & FASP & $T F A+A B C$ \\
\hline 14 & TCA & SDS & DTT & IAA & FASP & TFA + ABC \\
\hline
\end{tabular}




\section{A. Whole cell lysate sample preparation protocol study}
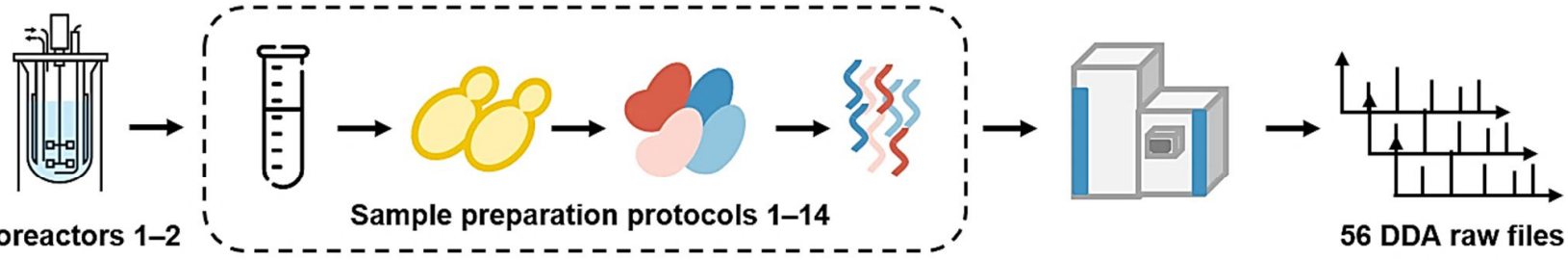

\section{B. Data processing workflow}

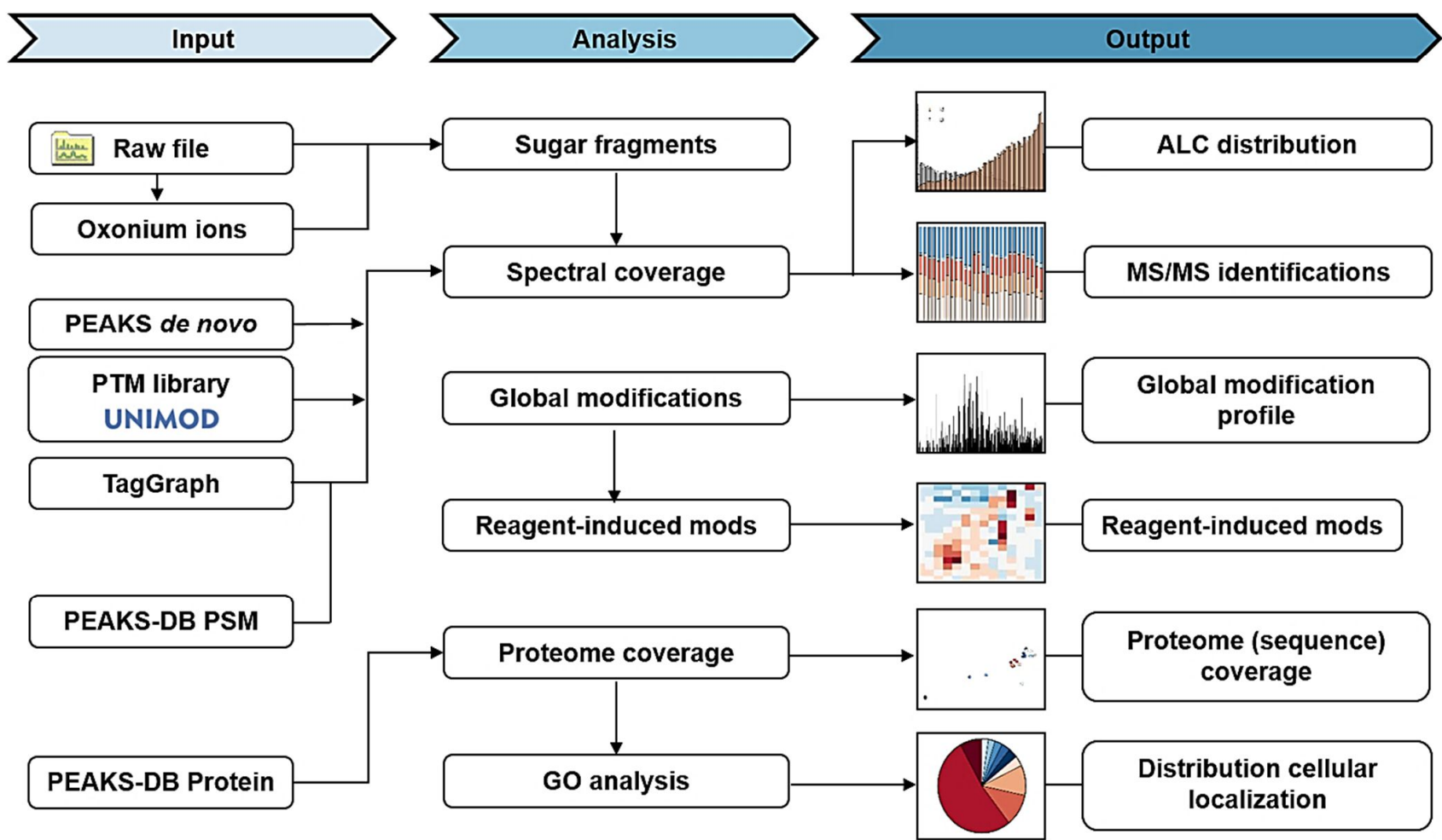

Figure 1: Whole cell lysate sample preparation study for yeast and established data processing pipeline. A. The employed study performed yeast cultivation in aerobic chemostats, sample collection and quenching, cell lysis by bead beating, protein extraction from cells and subsequent reduction and alkylation of the proteins. Proteins are then concentrated using protein precipitation or a filter-aided approach. Enriched proteins are subsequently digested using Trypsin, where the obtained peptides are subjected to solid phase extraction (SPE) to remove contaminants prior to shotgun proteomic analysis. B. The obtained MS/MS spectra were de novo sequenced using PEAKS and subsequently identified through database (PEAKS-DB) and unrestricted modification searches (TagGraph). A data analysis pipeline was established to determine the $\%$ of identified spectra, spectral quality, proteome and amino acid sequence coverage. Peptides identifications obtained by TagGraph were furthermore used to determine the modification profiles for every protocol. Finally, proteome were annotated with GO-terms to investigate the distribution according to 'cellular components'. 
A.
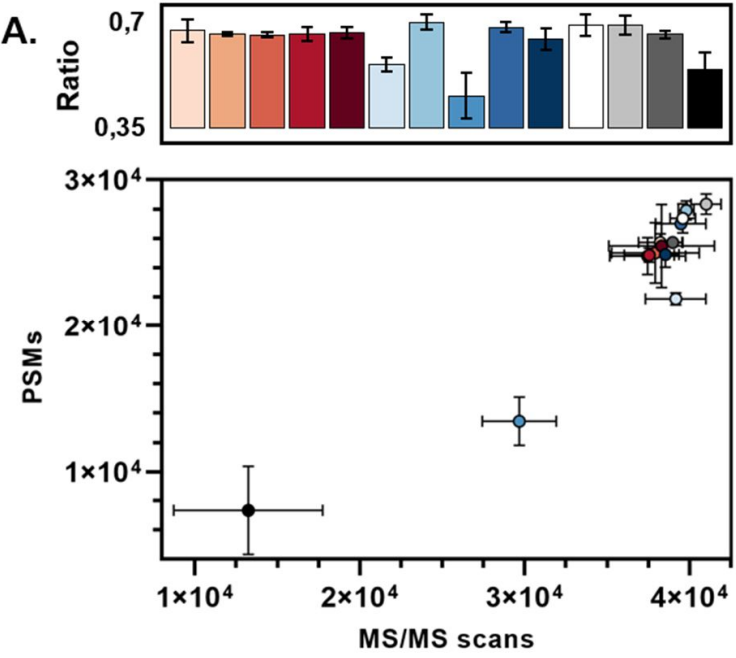

C.
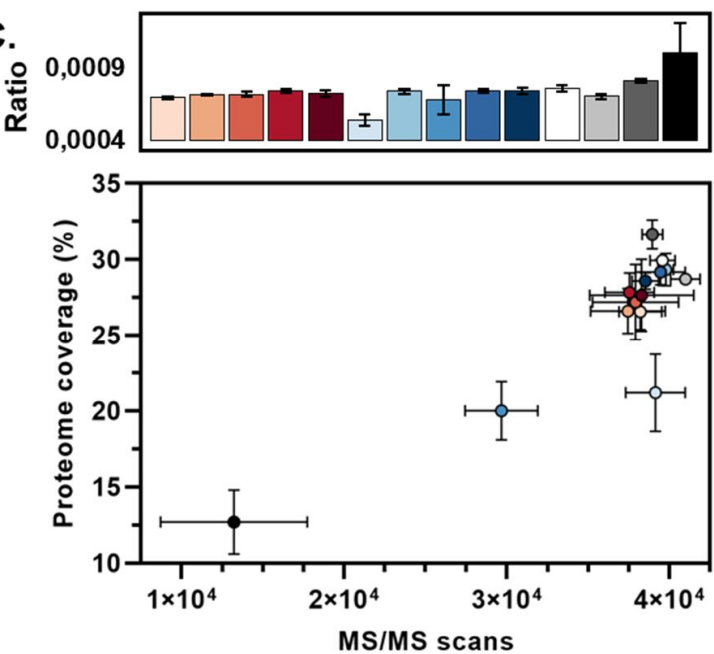

B.
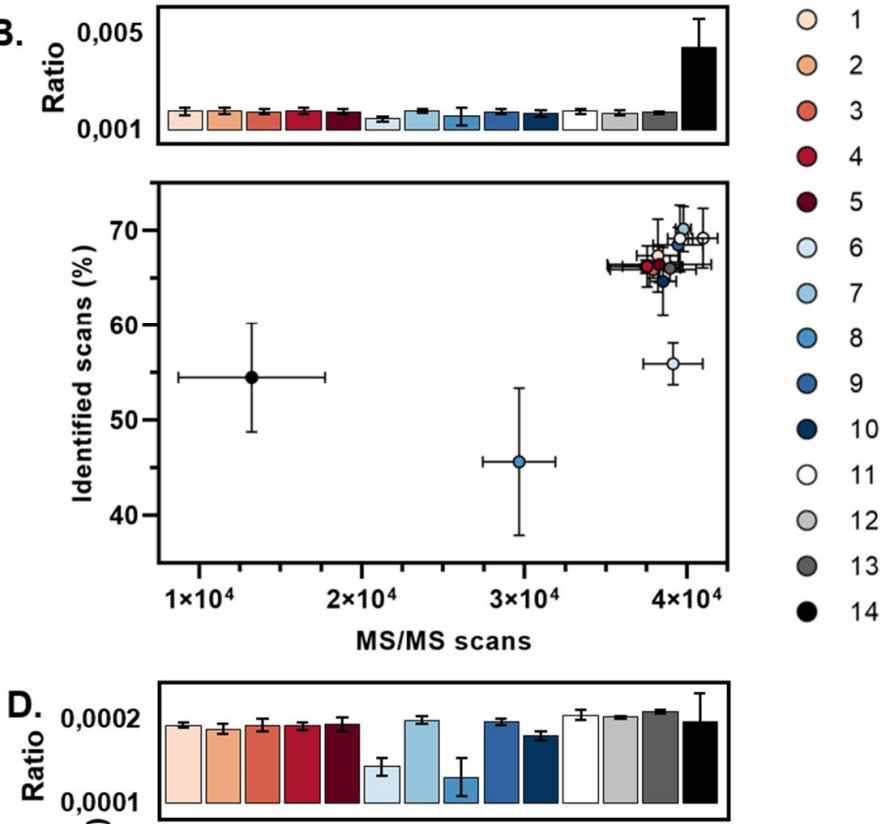

$\frac{0,000}{\circ}$

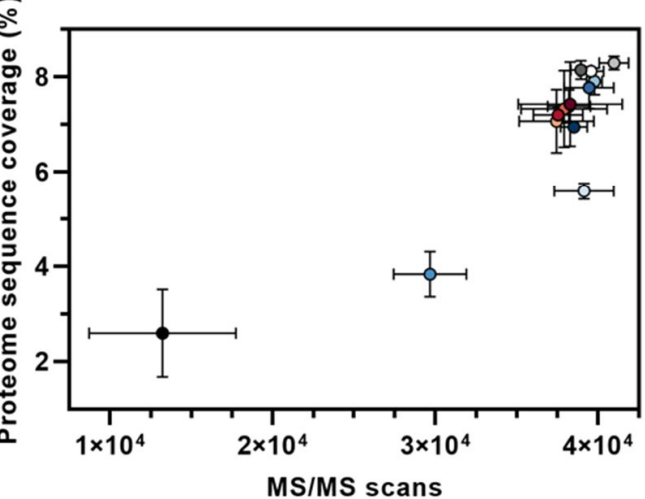

Figure 2: Achieved \% of identified fragmentation spectra, proteome and sequence coverage for the different sample preparation protocols. The number of Peptide Spectrum Matches (PSMs) (A); identified scans (\%) (B); proteome coverage (\%) (C) and proteome sequence coverage (D), were plotted against the number of MS/MS scans obtained per protocol. Each coloured circle represents the average of averages of two biological replicates with each 2 technical replicates $(2 \times 2)$, while the standard deviation is represented by the error bars. In addition, the bars depicted above each plot show the ratios of the acquired PSMs, identified scans and proteome (sequence) coverage vs. MS/MS scans for each protocol. The ratios are averages of two biological replicates with each 2 technical replicates (2x2), while the standard deviation is shown as error bars. The proteome coverage was calculated based on the identified proteins per protocol as a percentage of the total number of proteins in yeast (known ORFs). In addition, the proteome sequence coverage was calculated based on the identified amino acids in the proteomics experiments, as a percentage of the total proteome amino acid sequence (sequence coverage). 
A.

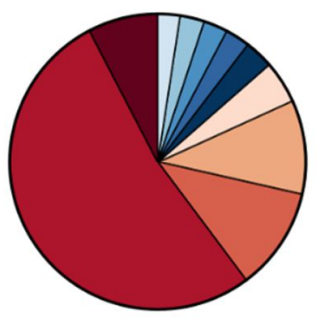

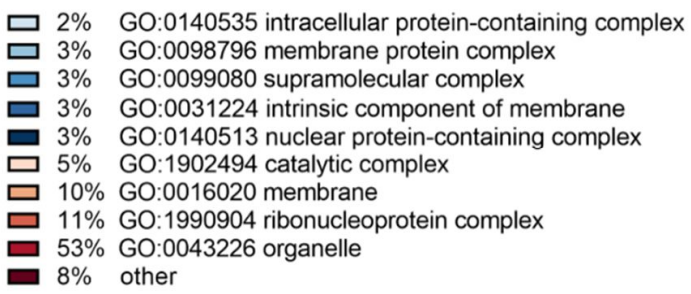

B.

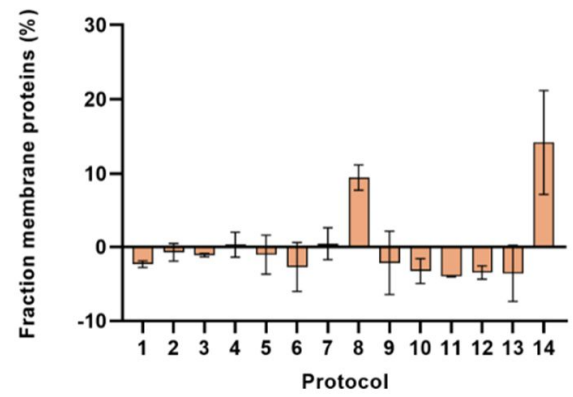

Figure 3: Distribution of Gene Ontology (GO) terms for identified proteins. A) Based on the classifications of GO annotation, the identified proteins (with at least 2 unique peptides) were categorized into cellular components, which is displayed in the pie chart for protocol 13 (biological replicate 1). Categories with GO terms less than 1\% are classified as 'other'. Here, the average of two biological replicates with each 2 technical replicates $(2 \times 2)$ is shown. B) The average fraction of identified membrane proteins across all protocols was $11 \%$. The deviations from the average are shown in percentage for each protocol (1-14).

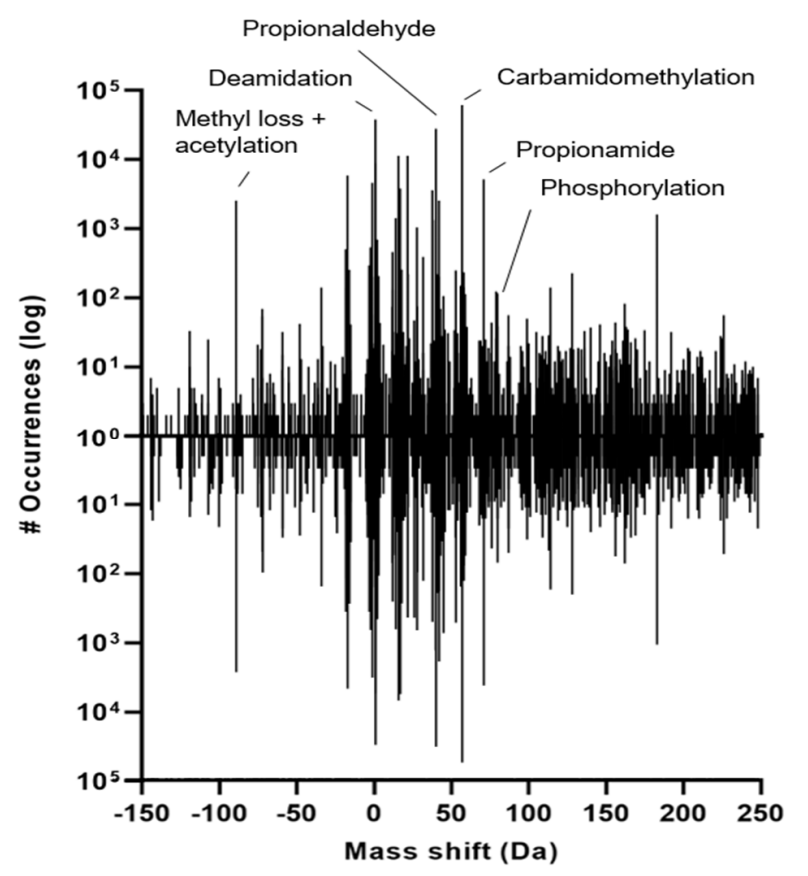

Figure 4: Sum of observed mass shifts in the different protocols using an unrestricted modification search approach (TagGraph). The number of occurrences corresponds to the number of peptide spectrum matches containing the mass deviation (log scale) for biological replicate 1 (upper histogram) and 2 (lower histogram). The total of both biological replicates show a highly comparable mass-shift profile. 


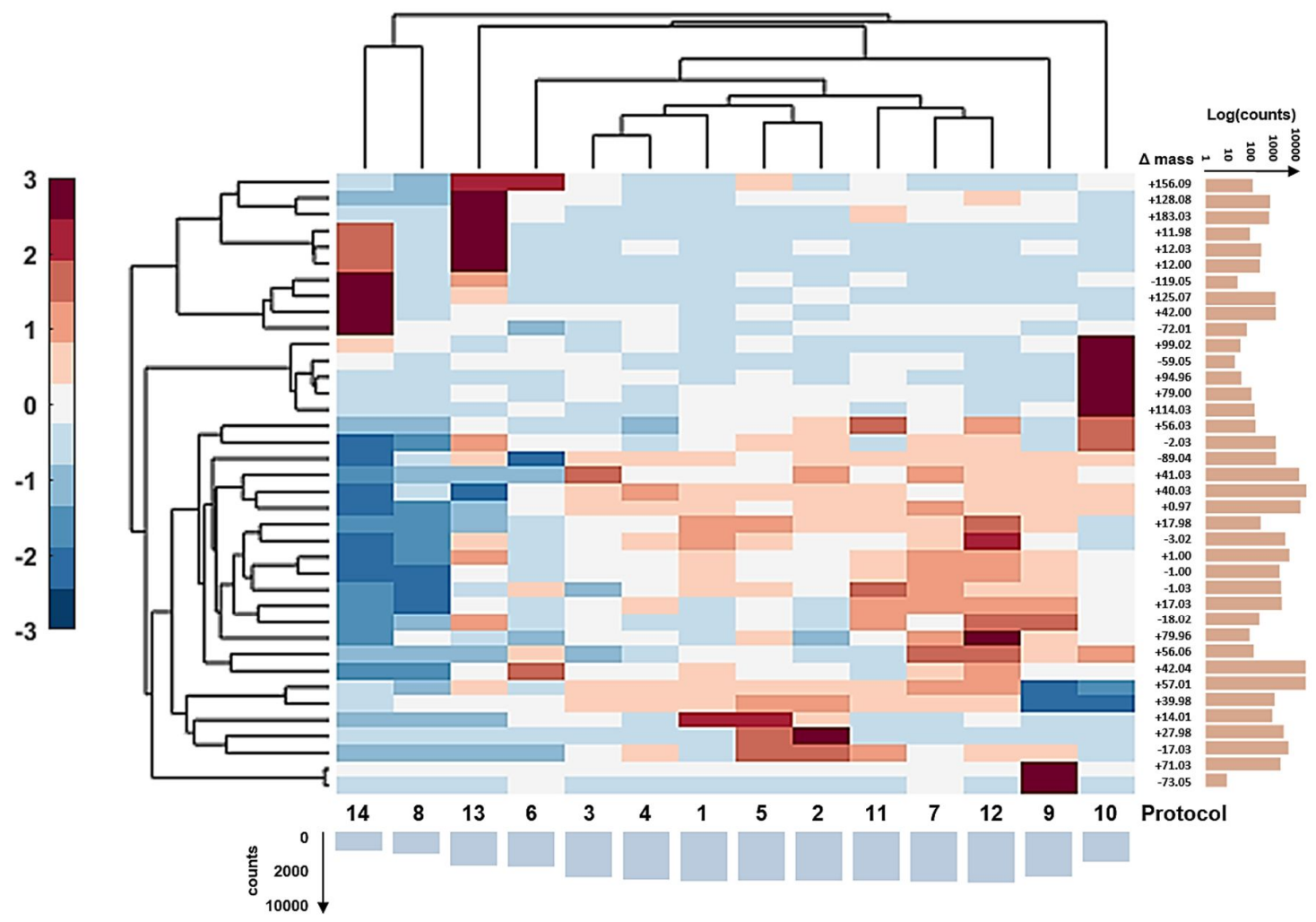

Figure 5: Clustered heat map of most prominent modifications identified by TagGraph across the different sample preparation protocols. Dendrogram and standardized clustered heat map of the relative change (\%) of mass shifts observed across the sample preparation protocols. The mass shifts found for each protocol originating from each technical and biological replicates $(2 \times 2)$, were averaged per sample and normalized to the number of obtained MS/MS spectra. The bars below the heat map show the total counts of modifications observed for each sample and the bars on the y-axis show the total counts of the respective mass shift (modification) across the investigated samples. 

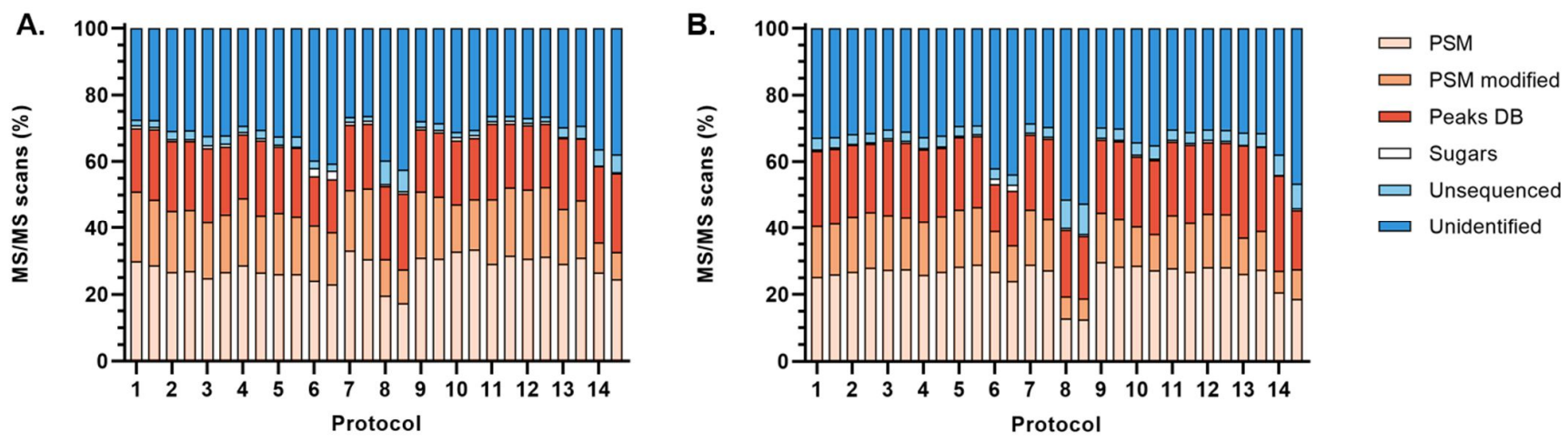

Figure 6: Overall spectral coverage obtained for the different sample preparation protocols. Allocation of total number of MS/MS spectra of each sample (1-14) into different categories: PSMs (TagGraph), PSMs modified (TagGraph), identified spectra by second search engine (PEAKS-DB), spectra containing oxonium fragments, 'unsequenced' and unidentified spectra for biological replicate 1 (A) and 2 (B).

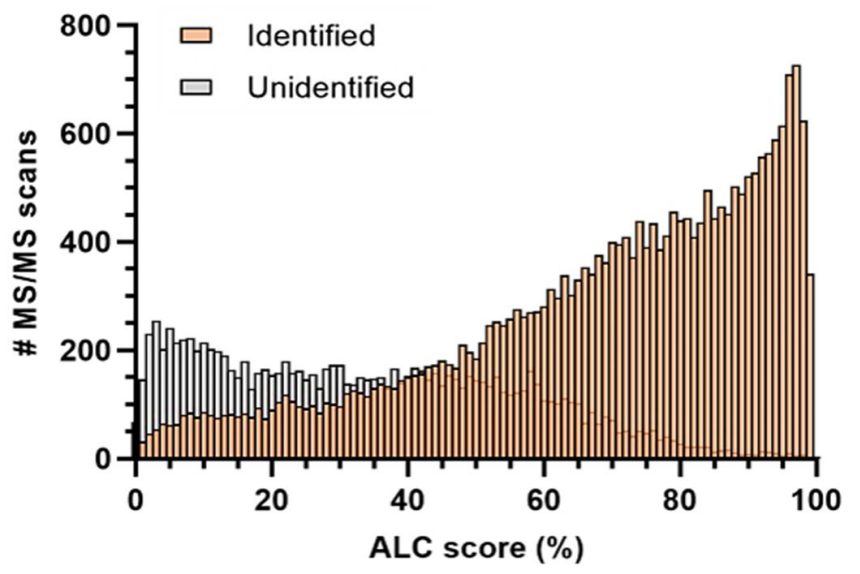

Figure 7: Assessing the spectral quality of the acquired fragmentation spectra. The Average Local Confidence (ALC) score distribution of identified (orange) and unidentified (grey) spectra, as shown for protocol 13 (biological replicate 1). In general, peptides with higher ALC score are more likely to provide a confident match during database search (because of the better fragment ion coverage of the peptide backbone, and the possibly 'cleaner' spectrum). The unidentified fraction from protocol 13 (grey) shows an ACL score distribution at predominantly lower numbers, indicating that the unidentified spectra largely derive from very low quality spectra, insufficient to provide a confident peptide sequence match. The fraction of higher ALC scoring spectra presumably derive from sequence variants or unexpected modifications not present in the database. Taxonomic impurities or 